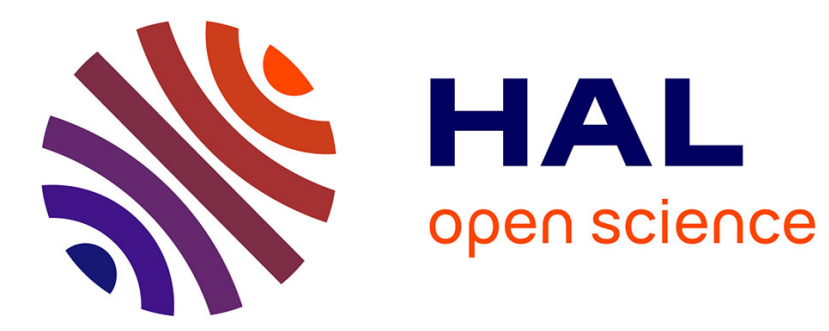

\title{
Pseudogap opening and formation of Fermi arcs as an orbital-selective Mott transition in momentum space
}

Michel Ferrero, Pablo Cornaglia, Lorenzo de Leo, Olivier Parcollet, Gabriel Kotliar, Antoine Georges

\section{To cite this version:}

Michel Ferrero, Pablo Cornaglia, Lorenzo de Leo, Olivier Parcollet, Gabriel Kotliar, et al.. Pseudogap opening and formation of Fermi arcs as an orbital-selective Mott transition in momentum space. Physical Review B: Condensed Matter and Materials Physics (1998-2015), 2009, 80 (6), 10.1103/PhysRevB.80.064501 . hal-02364045

\section{HAL Id: hal-02364045 \\ https://hal.science/hal-02364045}

Submitted on 12 Jul 2021

HAL is a multi-disciplinary open access archive for the deposit and dissemination of scientific research documents, whether they are published or not. The documents may come from teaching and research institutions in France or abroad, or from public or private research centers.
L'archive ouverte pluridisciplinaire HAL, est destinée au dépôt et à la diffusion de documents scientifiques de niveau recherche, publiés ou non, émanant des établissements d'enseignement et de recherche français ou étrangers, des laboratoires publics ou privés. 


\title{
Pseudogap opening and formation of Fermi arcs as an orbital-selective Mott transition in momentum space
}

\author{
Michel Ferrero, ${ }^{1,2}$ Pablo S. Cornaglia, ${ }^{3,1}$ Lorenzo De Leo, ${ }^{1}$ Olivier Parcollet, ${ }^{2}$ Gabriel Kotliar, ${ }^{4}$ and Antoine Georges ${ }^{1}$ \\ ${ }^{1}$ Centre de Physique Théorique, Ecole Polytechnique, CNRS, 91128 Palaiseau Cedex, France \\ ${ }^{2}$ Institut de Physique Théorique, CEA, IPhT, CNRS, URA 2306, 91191 Gif-sur-Yvette, France \\ ${ }^{3}$ Centro Atómico Bariloche and Instituto Balseiro, CNEA, CONICET, 8400 Bariloche, Argentina \\ ${ }^{4}$ Physics Department and Center for Materials Theory, Rutgers University, Piscataway NJ 08854, USA
}

(Dated: September 6, 2018)

\begin{abstract}
We present an approach to the normal state of cuprate superconductors which is based on a minimal cluster extension of dynamical mean-field theory. Our approach is based on an effective two-impurity model embedded in a self-consistent bath. The two degrees of freedom of this effective model can be associated to the nodal and antinodal regions of momentum space. We find a metal-insulator transition which is selective in momentum space: At low doping quasiparticles are destroyed in the antinodal region, while they remain protected in the nodal region, leading to the formation of apparent Fermi arcs. We compare our results to tunneling and angular-resolved photoemission experiments on cuprates. At very low energy, a simple description of this transition can be given using rotationally invariant slave bosons.
\end{abstract}

PACS numbers: 71.27.+a,71.30.+h,74.72.-h

\section{INTRODUCTION AND MOTIVATIONS}

The doping of a Mott insulator is a fundamental problem of condensed matter physics, which has attracted considerable attention in view of its relevance to the physics of cuprate superconductors 1 In the simplest Brinkman-Rice ${ }^{2}$ description, the doped metallic state is a Fermi liquid in which quasiparticles are formed with a heavy mass $m^{*} / m \sim 1 / \delta$ and a reduced weight $Z \sim \delta$ ( $\delta$ is the doping level). This physical picture can indeed be rationalized using the modern theoretical framework of dynamical mean-field theory (DMFT) $3,4,5$ DMFT, in its single-site version, is applicable when spatial correlations are weak, which is favored by high dimensionality and strong competing (e.g. orbital) fluctuations.

In cuprates however, which are two-dimensional materials with low orbital degeneracy, it was pointed out long ago by Anderson in a seminal paper ${ }^{1}$ that the antiferromagnetic superexchange $(J)$ plays a key role, leading to strong short-range correlations associated with singlet formation (valence bonds) between nearest-neighbor lattice sites. Slave boson mean-field theories $6,7,8,9,10$ as well as projected variational wave-functions, ${ }^{11,12}$ provide simple theoretical frameworks to incorporate this effect, modifying the Brinkman-Rice picture at small doping $\delta \lesssim J / t$ and leading in particular to a finite effective mass of quasiparticles $m^{*} / m \sim 1 /(J / t+\delta)$. This is indeed consistent with observations in cuprates, in which only a moderate enhancement of the effective mass is observed.

However, both single-site DMFT and simple variational or slave-boson mean-field theories share a common feature, namely that the characteristic energy (or temperature) scale below which coherent quasiparticles are formed is uniform along the Fermi surface, and of order $\delta t$ at small doping levels. This is clearly inconsistent with experimental observations in underdoped cuprates. In- deed, these materials are characterized by a strong differentiation of quasiparticle properties in momentum space, a phenomenon which is key to their unusual normal-state properties. In underdoped cuprates, coherent quasiparticle excitations are suppressed in the antinodal regions, around momenta $(0, \pi)$ and $(\pi, 0)$ of the Brillouin zone (BZ), and a pseudogap appears below a characteristic temperature scale (which decreases as the doping level is increased). Instead, reasonably coherent quasiparticles are preserved in the nodal regions around $(\pi / 2, \pi / 2)$. The signature of this phenomenon in angular-resolved photoemission spectroscopy (ARPES) is the formation of Fermi 'arcs' in the underdoped regime, defined as the regions of momentum space where the spectral function is intense at low excitation energy (see e.g. Ref. 13 for a review). Suppression of quasiparticle coherence in the antinodal regions is also apparent from other spectroscopies, such as electronic Raman scattering 14,15,16 (with the $\mathrm{B}_{1 g}$ and $\mathrm{B}_{2 g}$ channels associated with antinodes and nodes, respectively) or quasiparticle interference patterns obtained by scanning tunneling microscopy. 17

Momentum-space differentiation and the nodal/antinodal dichotomy is therefore an outstanding challenge for theories of strongly-correlated electrons. Various lines of attack to this problem have been taken. At intermediate and strong coupling, and apart from the extremely low-doping region, correlation lengths are expected to be short (as also supported by experimental observations). Hence, it is appropriate in this regime to take into account short-range correlations within cluster extensions of the DMFT framework. Such investigations have been quite successful (for reviews, see e.g. Refs. 4, 18, 19). Most studies have considered clusters of at least four sites (a plaque-

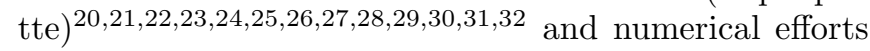
have been devoted to increasing the cluster size in order to improve momentum-resolution and to advance toward 
an understanding of the two-dimensional case $\mathrm{3}^{\underline{33}}$

In this article, we follow a different route, looking for a description based on the minimal cluster able to successfully describe momentum-space differentiation together with Mott physics ${ }^{34}$ We find that a two-site cluster is sufficient to achieve this goal on a qualitative level, and to a large extent on a quantitative level, when compared to larger cluster calculations. Our approach is based on a division of the BZ into two patches, one containing in particular the $(\pi / 2, \pi / 2)$ momentum and the other one in particular the $(\pi, 0)$ and $(0, \pi)$ momenta. A mapping onto a two-impurity Anderson model with self-consistent hybridization functions is made, following a generalization of the dynamical cluster-approximation construction (very similar results are actually obtained within a cellular-DMFT self-consistency condition). The self-energies associated with the 'nodal' and 'antinodal' patches are shown to correspond to the bonding (even) and antibonding (odd) orbitals of the self-consistent impurity model, respectively. This allows us to construct a 'valence-bond dynamical mean-field theory' (VBDMFT) ${ }^{34}$ of nodal/antinodal differentiation, in which this phenomenon is associated with the distinct properties of the orbitals associated with different regions of momentum space.

One of our central results is that, below a critical value of the doping level $\delta_{c} \simeq 16 \%$, the 'nodal' (bonding) orbital remains metallic while the 'antinodal' (antibonding) orbital displays a pseudogap. Correspondingly, the scattering rate associated with the nodal orbital is suppressed in the pseudogap state, while the antinodal orbital scattering rate increases as the doping level is reduced. Hence, nodal/antinodal differentiation corresponds in this minimal description to an orbital differentiation, a momentum-space analogue $e^{35}$ of the orbitalselective Mott transition which has been extensively discussed recently in the different context of transitionmetal oxides with several active orbitals $\underline{36}$ The suppressed coherence of antinodal quasiparticles clearly originates, in our description, from Mott physics affecting antinodal regions in a dramatic manner while nodal regions remain comparatively protected.

A definite advantage and important motivation for building a minimal description based on the smallest possible cluster is to advance our qualitative understanding. Since the theory is based on a two-site Anderson model, results can be interpreted in terms of valence-bond singlet formation and linked to the well-documented competition between singlet-formation and individual Kondo screening. ${ }^{37,38}$ The two-impurity Kondo (or Anderson) model is the simplest model which captures this competition. However our findings show that, in contrast to the two-impurity model with a fixed hybridization to the conduction-electron bath, the additional self-consistency of the bath which is central to dynamical mean-field constructions brings in novel aspects. Indeed, the critical point encountered at a finite doping $\delta_{c}$ is found only in the lattice model involving self-consistent baths, while it is replaced by a crossover in the non self-consistent twoimpurity Anderson model.

This article is organized as follows. In Sec. IA we specify the two-dimensional Hubbard model under consideration and describe the BZ patching and VB-DMFT mapping onto a self-consistent two-orbital model. Then, we briefly review the two main techniques that we have used for the solution of this problem: the strong-coupling continuous-time quantum Monte Carlo algorithm ${ }^{39.40}$ (Sec. IIB) and the (semianalytical) rotationally-invariant slave-boson approximation ${ }^{41}$ (Sec. IIC). Sec. III is devoted to a detailed presentation of the orbital-selective transition and of its physical relevance to nodal/antinodal differentiation. In particular, the frequency dependence of the self-energies and spectral functions on the real axis are presented and interpreted. In Sec. IV these results are contrasted to the physics of a two-impurity problem with non selfconsistent baths, in connection with the Kondo to RKKY (singlet) crossover observed there. Finally, in Sec. VA. the issue of momentum-space reconstruction is considered, and the connection to the formation of 'Fermi arcs' is discussed. For the sake of clarity and completeness, some technical aspects of our work are discussed more in detail in appendices.

\section{THEORETICAL FRAMEWORK}

\section{A. Model and valence-bond dynamical mean-field theory}

We study the Hubbard model on a square lattice, with hopping between nearest-neighbor $t$ and next-nearestneighbor sites $t^{\prime}$. The corresponding Hamiltonian is given by

$$
\begin{aligned}
H & =\sum_{\mathbf{k}, \sigma=\uparrow, \downarrow} \varepsilon_{\mathbf{k}} c_{\sigma \mathbf{k}}^{\dagger} c_{\sigma \mathbf{k}}+U \sum_{i} n_{i \downarrow} n_{i \uparrow} \\
\varepsilon_{\mathbf{k}} & =-2 t\left(\cos \left(\mathbf{k}_{x}\right)+\cos \left(\mathbf{k}_{y}\right)\right)-4 t^{\prime} \cos \left(\mathbf{k}_{x}\right) \cos \left(\mathbf{k}_{y}\right) .
\end{aligned}
$$

In the following, we use $U / t=10$ and $t^{\prime} / t=-0.3$, which are values commonly used for modeling hole-doped cuprates in a single-band framework. All energies (and temperatures) are expressed in units of $D=4 t=1$, and the doping is denoted by $\delta$. We restrict ourselves to paramagnetic normal phases.

In this paper, we focus on a two-site dynamical cluster approximation (DCA) $\stackrel{18}{=}$ For completeness, we recall the DCA construction in Appendix A The principle of the DCA approximation is to cut the Brillouin zone into patches and approximate the self-energy as a piecewise constant function on the patches. The many-body problem can then be solved using an effective self-consistent multiple quantum impurity model. A priori, there is some arbitrariness in the choice of patches in DCA. In this paper, we exploit this freedom in order to separate the nodal and the antinodal region into two patches, so 
that the properties of each region will be described by one orbital of the effective impurity model. More precisely, we choose the minimal set of two patches of equal area $P_{+}$and $P_{-}$represented in Fig. 1) $P_{+}$is a central square centered at momentum $(0,0)$ and containing the nodal region; the complementary region $P_{-}$extends to the edge of the BZ and contains in particular the antinodal region and the $(\pi, \pi)$ momentum. On Fig. 2, we also present the partial density of state of both patches.

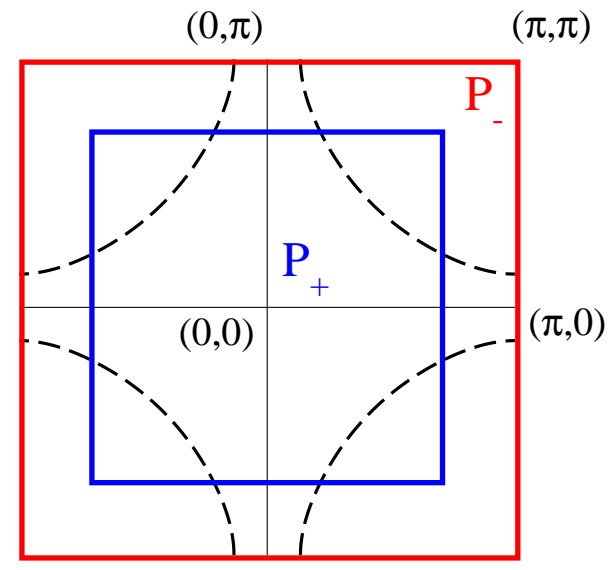

FIG. 1: (Color online) The Brillouin zone is divided into two patches $P_{+}$(inside the inner blue square) and $P_{-}$(between the two squares). The dotted line is the free $(U=0)$ Fermi surface at $\delta=0.1$ for $t^{\prime} / t=-0.3$. $P_{+}$(resp. $P_{-}$) encloses the nodal (resp. antinodal) region.

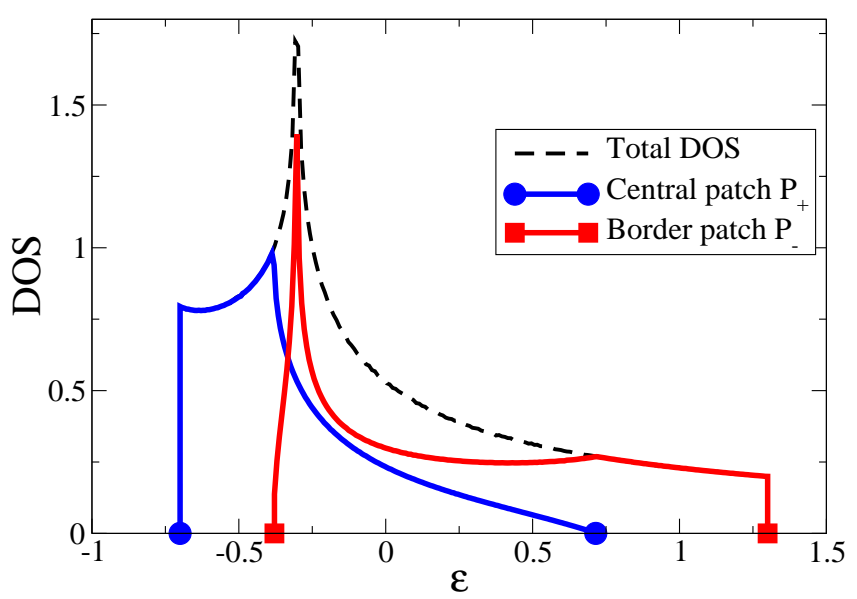

FIG. 2: (Color online) Partial density of states of the two patches $P_{+}$(solid blue curve with circles) and $P_{-}$(solid red curve with squares), and total density of states (dashed curve); $t^{\prime} / t=-0.3$.

It is important to check that the main qualitative results of our approach are independent of the precise shape of the patches. We will discuss this point in Sec. IIIE and show that indeed our results are qualitatively similar for a family of patches in which the $P_{+}$patch encloses a variable part of the bare Fermi surface around the nodal point. Moreover, we have also considered another cluster method, cellular-DMFT (CDMFT),$\underline{4,18}$ and obtained qualitatively similar results. Because two-site CDMFT breaks the lattice square symmetry, we focus here on a generalized DCA approach.

Following the DCA construction (see also Appendix (A), we associate a momentum-independent selfenergy $\Sigma_{ \pm}(\omega)$ to each patch of the Brillouin zone. This self-energy is then identified with the Fourier transform of the cluster self-energy of a two-site cluster of Anderson impurities embedded in a self-consistent bath. This two-site Anderson impurity model is given by

$$
\begin{gathered}
S_{\mathrm{eff}}=-\iint_{0}^{\beta} d \tau d \tau^{\prime} \sum_{\substack{a, b=1,2 \\
\sigma=\uparrow, \downarrow}} c_{a \sigma}^{\dagger}(\tau) G_{0, a b}^{-1}\left(\tau, \tau^{\prime}\right) c_{b \sigma}\left(\tau^{\prime}\right) \\
+\int_{0}^{\beta} d \tau U \sum_{a=1,2} n_{a \downarrow} n_{a \uparrow}(\tau) \\
G_{0 a b}^{-1}\left(i \omega_{n}\right)=\left(i \omega_{n}+\mu\right) \delta_{a b}-\bar{t}\left(1-\delta_{a b}\right)-\Delta_{a b}\left(i \omega_{n}\right),
\end{gathered}
$$

where $a, b=1,2$ is the site index, $U$ is the on-site interaction, $\Delta$ is the hybridization function with a local component $\Delta_{11}(\omega)=\Delta_{22}(\omega)$ and an inter-site one $\Delta_{12}(\omega)$. We choose a convention in which the hybridization $\Delta$ vanishes at infinite frequencies and therefore denote the constant term separately $(\bar{t})$. Since we restrict ourselves to paramagnetic solutions, we dropped the spin dependence of $G_{0}, \Delta$ and $\bar{t}$. The self-consistency condition determines both $\Delta$ and $\bar{t}$ and is written in the Fourier space of the cluster, which in this case reduces to the even and odd orbital combinations $c_{ \pm \sigma}^{\dagger}=\left(c_{1 \sigma}^{\dagger} \pm c_{2 \sigma}^{\dagger}\right) / \sqrt{2}$ :

$$
\begin{aligned}
\Sigma_{K}\left(i \omega_{n}\right) & =G_{0 K}\left(i \omega_{n}\right)^{-1}-G_{K}\left(i \omega_{n}\right)^{-1} \\
G_{K}\left(i \omega_{n}\right) & =\sum_{\mathbf{k} \in P_{K}} \frac{1}{i \omega_{n}+\mu-\varepsilon_{\mathbf{k}}-\Sigma_{K}\left(i \omega_{n}\right)} .
\end{aligned}
$$

In this expression, momentum summations are normalized to unity within each patch, and the index $K= \pm$ refers both to the inner/outer patch index and to the even/odd orbital combinations of the two-impurity problem. $\bar{t}$ is determined by the $1 / \omega^{2}$ expansion of the previous equations, leading to

$$
\bar{t}=\sum_{\mathbf{k} \in P_{+}} \varepsilon_{\mathbf{k}}=-\sum_{\mathbf{k} \in P_{-}} \varepsilon_{\mathbf{k}}
$$

The impurity model has the same local interaction as the original lattice model: This is a consequence of the fact that both patches have equal surface (see Appendix A).

As usual in the DMFT problems, the quantum impurity model (3) can be rewritten in a Hamiltonian form, i.e. as the Hamiltonian for a dimer coupled to a selfconsistent bath

$$
H=H_{\text {dimer }}+H_{\text {bath }},
$$


where $H_{\text {dimer }}$ can be written in the 1,2 basis as

$$
H_{\text {dimer }} \equiv \sum_{\substack{a, b=1,2 \\ \sigma=\uparrow, \downarrow}} c_{a \sigma}^{\dagger}\left(\bar{t}\left(1-\delta_{a b}\right)+\varepsilon_{0} \delta_{a b}\right) c_{b \sigma}+\sum_{a=1,2} U n_{a \downarrow} n_{a \uparrow},
$$

where $\varepsilon_{0} \equiv-\mu$. Alternatively, $H_{\text {dimer }}$ can be written in the even/odd basis where the hybridization is diagonal

$$
\begin{gathered}
H_{\text {dimer }}=\sum_{\substack{s= \pm \sigma=\uparrow, \downarrow}} c_{s \sigma}^{\dagger}\left(s \bar{t}+\varepsilon_{0}\right) c_{s \sigma}+ \\
\frac{U}{2} \sum_{\substack{s= \pm \bar{s}=-s}}\left(n_{s \uparrow} n_{s \downarrow}+n_{s \uparrow} n_{\bar{s} \downarrow}+c_{s \uparrow}^{\dagger} c_{s \downarrow}^{\dagger} c_{\bar{s} \downarrow} c_{\bar{s} \uparrow}+c_{s \uparrow}^{\dagger} c_{\bar{s} \downarrow}^{\dagger} c_{s \downarrow} c_{\bar{s} \uparrow}\right) .
\end{gathered}
$$

Note that, since we will be solving the quantum impurity model using continuous-time quantum Monte Carlo and rotationally-invariant slave-boson methods, which work within the action formalism, we will not need the explicit form of the bath term $H_{\text {bath }}$.

\section{B. Continuous-time Monte Carlo}

A numerically exact solution of the self-consistent two-impurity problem is obtained using continuous-time quantum Monte Carlo (CTQMC) ${ }^{39,40}$ which sums the perturbation theory in $\Delta_{a b}\left(i \omega_{n}\right)$ on the Matsubara axis. The partition function of the impurity model

$$
Z=\int \mathcal{D} c^{\dagger} \mathcal{D} c \exp \left(-S_{\text {eff }}\right)
$$

is expanded in powers of the hybridization $\Delta$, leading to

$$
\begin{array}{r}
Z=\sum_{n \geq 0} \frac{1}{n !} \int \prod_{i=1}^{n} d \tau_{i} d \tau_{i}^{\prime} \sum_{\substack{a_{i}= \pm \sigma_{i}=\uparrow, \downarrow}} \operatorname{det}_{1 \leq i, j \leq n}\left[\Delta_{a_{i}, a_{j}}\left(\tau_{i}-\tau_{j}^{\prime}\right)\right] \times \\
\operatorname{Tr}\left(\mathcal{T} e^{-\beta H_{\text {dimer }}} \prod_{i=1}^{n} c_{a_{i} \sigma_{i}}^{\dagger}\left(\tau_{i}\right) c_{a_{i} \sigma_{i}}\left(\tau_{i}^{\prime}\right)\right),
\end{array}
$$

where $\mathcal{T}$ is time ordering and $H_{\text {dimer }}$ is given by (9).

The partition function is then sampled with the Metropolis algorithm, where a configuration of size $n$ is given by the $n$ indices $a_{i}, \sigma_{i}$ and times $\tau_{i}, \tau_{i}^{\prime}$ of the $c^{\dagger}, c$ operators. The Monte Carlo probability of a configuration is given by the absolute value of the product of the trace and the determinant term. The Green's function is then accumulated using the formula obtained by differentiating $Z$ with respect to $\Delta, \underline{39}, \underline{40}$

Another quantity of interest is the relative weight of multiplets of the dimer problem, which we will discuss in detail in Sec. IV] It can be measured, following Ref. 40, by their relative contribution to the trace: if $\Gamma$ is a multiplet of $H_{\text {dimer }}$, we define the CTQMC statistical weight of $\Gamma$ as

$p_{\Gamma}^{\mathrm{QMC}} \equiv\left\langle\frac{\left|\left\langle\Gamma\left|\mathcal{T} e^{-\beta H_{\mathrm{dimer}}} \mathcal{C}_{n}\left(a_{i}, \sigma_{i}, \tau_{i}, \tau_{i}^{\prime}\right)\right| \Gamma\right\rangle\right|}{\sum_{\Gamma}\left|\left\langle\Gamma\left|\mathcal{T} e^{-\beta H_{\mathrm{dimer}}} \mathcal{C}_{n}\left(a_{i}, \sigma_{i}, \tau_{i}, \tau_{i}^{\prime}\right)\right| \Gamma\right\rangle\right|}\right\rangle$

$\mathcal{C}_{n}\left(a_{i}, \sigma_{i}, \tau_{i}, \tau_{i}^{\prime}\right) \equiv \prod_{i=1}^{n} c_{a_{i} \sigma_{i}}^{\dagger}\left(\tau_{i}\right) c_{a_{i} \sigma_{i}}\left(\tau_{i}^{\prime}\right)$,

where $\langle A\rangle_{\mathrm{QMC}}$ denotes the Monte Carlo averaging over the configurations (labeled by $n$ and $\left\{a_{i}, \sigma_{i}, \tau_{i}, \tau_{i}^{\prime}\right\}$ ).

In this simple two-impurity model in the paramagnetic phase, the symmetry between the two sites allows to factorize the determinants: $: 39,40 \Delta$ is indeed diagonal in the \pm basis. We also note that the Monte Carlo sign is one in this problem. Due to the efficiency of this algorithm, we can routinely do a few millions Monte Carlo sweeps and obtain high-quality data in imaginary time. We then perform the continuation to the real axis using a simple Padé method ${ }^{42}$ (see also Sec. IIIB and Appendix B).

\section{Rotationally-invariant slave bosons}

Slave-boson (SB) methods (see e.g. Ref. 41,43, 44, 45) provide a simplified description of the low-energy excitations in strongly-correlated electron systems. The general idea is to enlarge the original Hilbert space to a set of states which involve both ('slave') bosonic and ('quasiparticle') fermionic variables. The bosonic variables are introduced in such a way that the interaction term becomes a simple quadratic form in terms of the slave bosons. The physical Hilbert space is recovered by imposing a (quadratic) constraint relating the slave bosons to the fermions. Mean-field (approximate) solutions can be obtained by looking for saddle points at which the bosons are condensed and at which the constraint is only satisfied on average. At such a saddle point, the fermionic variables can be interpreted as the low-energy quasiparticles of the original problem. A very simple form of the self-energy is obtained, containing only a constant term and a term linear in frequency. This should be interpreted as a simplified low-energy description of the system (i.e. as the first two terms in a lowfrequency expansion of the self-energy).

Because of the simplicity of this physical interpretation, slave-boson methods are a very complementary tool to fully numerical algorithms such as the one reviewed in the previous section. For this reason, we have also considered a SB mean-field solution of the VB-DMFT equations, which should be viewed as a simplified description of the low-energy physics. The full numerical solution (obtained with CTQMC) has of course a rich frequency dependence but, as we shall see, the SB approximation compares very well to the numerical results at low energy.

To be specific, we use a slave-boson mean-field as an approximate 'impurity solver' of the effective twoimpurity problem coupled to self-consistent baths, within 
TABLE I: Eigenstates of the dimer. The quantum numbers for charge, spin, and parity are given. The last column shows the slave bosons for the description of the eigenstates in the RISB formalism. States 10 and 11 have all their quantum numbers equal and form a $2 \times 2$ block. The ground state is number 10 which is the antiferromagnetic singlet of even parity and has an energy $E_{10}=2 \varepsilon_{0}+\frac{1}{2}\left(U-\sqrt{16 t^{2}+U^{2}}\right) \simeq 2 \varepsilon_{0}-4 \bar{t}^{2} / U$. Here $\ell_{ \pm}=U \pm \sqrt{16 t^{2}+U^{2}} / 4 \bar{t}$ and $\mathcal{N}_{ \pm}=2+2 \ell_{ \pm}^{2}$.

\begin{tabular}{|c|c|c|c|c|c|c|c|}
\hline No. & Label (cf. Fig. 15) & Eigenstate & $n_{\uparrow}$ & $n_{\downarrow}$ & Parity & S & Boson \\
\hline 1 & $\mathrm{E}$ & $|0,0\rangle$ & 0 & 0 & + & 0 & $\phi_{1,1}$ \\
\hline 2 & $1+\left(S_{z}=+1 / 2\right)$ & $\frac{1}{\sqrt{2}}(|0, \uparrow\rangle+|\uparrow, 0\rangle)$ & 1 & 0 & + & $1 / 2$ & $\phi_{2,2}$ \\
\hline 3 & & $\frac{1}{\sqrt{2}}(|0, \uparrow\rangle-|\uparrow, 0\rangle)$ & 1 & 0 & - & $1 / 2$ & $\phi_{3,3}$ \\
\hline 4 & $1+\left(S_{z}=-1 / 2\right)$ & $\frac{1}{\sqrt{2}}(|0, \downarrow\rangle+|\downarrow, 0\rangle)$ & 0 & 1 & + & $1 / 2$ & $\phi_{4,4}$ \\
\hline 5 & & $\frac{1}{\sqrt{2}}(|0, \downarrow\rangle-|\downarrow, 0\rangle)$ & 0 & 1 & - & $1 / 2$ & $\phi_{5,5}$ \\
\hline 6 & $\mathrm{~T}\left(S_{z}=+1\right)$ & $|\uparrow, \uparrow\rangle$ & 2 & 0 & - & 1 & $\phi_{6,6}$ \\
\hline 7 & $\mathrm{~T}\left(S_{z}=-1\right)$ & $|\downarrow, \downarrow\rangle$ & 0 & 2 & - & 1 & $\phi_{7,7}$ \\
\hline 8 & $\mathrm{~T}\left(S_{z}=0\right)$ & $\frac{1}{\sqrt{2}}(|\uparrow, \downarrow\rangle+|\uparrow, \downarrow\rangle)$ & 1 & 1 & - & 1 & $\phi_{8,8}$ \\
\hline 9 & & $\frac{1}{\sqrt{2}}(|0, \uparrow \downarrow\rangle-|\uparrow \downarrow, 0\rangle)$ & 1 & 1 & - & 0 & $\phi_{9,9}$ \\
\hline 10 & S & $\frac{1}{\sqrt{\mathcal{N}_{-}}}\left(-|\uparrow, \downarrow\rangle+\ell_{-}|\uparrow \downarrow, 0\rangle+\ell_{-}|0, \uparrow \downarrow\rangle+|\downarrow, \uparrow\rangle\right)$ & 1 & 1 & + & 0 & $\phi_{10,10} ; \phi_{10,11}$ \\
\hline 11 & & $\frac{1}{\sqrt{\mathcal{N}_{+}}}\left(-|\uparrow, \downarrow\rangle+\ell_{+}|\uparrow \downarrow, 0\rangle+\ell+|0, \uparrow \downarrow\rangle+|\downarrow, \uparrow\rangle\right)$ & 1 & 1 & + & 0 & $\phi_{11,10} ; \phi_{11,11}$ \\
\hline 12 & & $\frac{1}{\sqrt{2}}(|\uparrow \downarrow, \uparrow\rangle+|\uparrow, \uparrow \downarrow\rangle)$ & 2 & 1 & + & $1 / 2$ & $\phi_{12,12}$ \\
\hline 13 & & $\frac{1}{\sqrt{2}}(|\uparrow \downarrow, \uparrow\rangle-|\uparrow, \uparrow \downarrow\rangle)$ & 2 & 1 & - & $1 / 2$ & $\phi_{13,13}$ \\
\hline 14 & & $\frac{1}{\sqrt{2}}(|\uparrow \downarrow, \downarrow\rangle+|\downarrow, \uparrow \downarrow\rangle)$ & 1 & 2 & + & $1 / 2$ & $\phi_{14,14}$ \\
\hline 15 & & $\frac{1}{\sqrt{2}}(|\uparrow \downarrow, \downarrow\rangle-|\downarrow, \uparrow \downarrow\rangle)$ & 1 & 2 & - & $1 / 2$ & $\phi_{15,15}$ \\
\hline 16 & & $|\uparrow \downarrow, \uparrow \downarrow\rangle$ & 2 & 2 & + & 0 & $\phi_{16,16}$ \\
\hline
\end{tabular}

the self-consistency iterative loop of VB-DMFT. We use the recently introduced 'rotationally-invariant' slaveboson formalism 41 (RISB), which generalizes the original construction of Kotliar and Ruckenstein 45 to multiorbital systems in a way which respects all symmetries of the Hamiltonian (see also Refs. 46, 47). To define the slave-boson variables, we consider the ('molecular') eigenstates $|\Gamma\rangle$ of the Hamiltonian (9) describing the isolated 2-site cluster in the absence of the baths. For definiteness, these 16 states and their quantum numbers are listed in Table I] In the original formulation of Kotliar and Ruckenstein, ${ }^{45}$ a slave boson $\phi_{\Gamma}$ is introduced for each molecular eigenstate. However, this breaks rotational invariance in spin and orbital space, and results into difficulties when saddle-point solutions are considered. For example, nonequivalent saddle-point solutions would be found depending on whether the Hamiltonian is expressed in the $(1,2)$-basis (corresponding to sites) or in the $(+,-)$ basis corresponding to the even and odd orbital. To avoid this problem, the RISB ${ }^{41}$ formalism introduces a matrix of slave-boson amplitudes, $\left\{\phi_{\Gamma \Gamma^{\prime}}\right\}$. The first index $(\Gamma)$ is associated with each molecular eigenstate in the physical Hilbert space of the dimer. The second index $\left(\Gamma^{\prime}\right)$ corresponds to a state of the quasiparticle fermionic variables (with the same fermionic content as the corresponding physical state). Symmetry considerations allow for a drastic reduction of the number of bosons to be considered in practice: Only the $\phi_{\Gamma \Gamma^{\prime}}$ such that both states have identical quantum numbers take non-zero values at the saddle point. For the problem at hand, this leaves 18 boson amplitudes in total, all scalars except for a $2 \times 2$ block in the two-particle sector with total spin $S=0$ and even parity (Table $\amalg$ ). Other bosons turn out to be zero at the mean-field level.

To exclude the nonphysical states we impose the following set of constraints

$$
\begin{aligned}
& \sum_{\Gamma \Gamma^{\prime}} \phi_{\Gamma \Gamma^{\prime}}^{\dagger} \phi_{\Gamma \Gamma^{\prime}}=1 \\
& \sum_{\Gamma \Gamma_{1}^{\prime} \Gamma_{2}^{\prime}} \phi_{\Gamma \Gamma_{1}^{\prime}}^{\dagger} \phi_{\Gamma \Gamma_{2}^{\prime}}\left\langle\Gamma_{2}^{\prime}\left|f_{\alpha}^{\dagger} f_{\beta}\right| \Gamma_{1}^{\prime}\right\rangle=f_{\alpha}^{\dagger} f_{\beta}, \quad \forall \alpha, \beta,(14)
\end{aligned}
$$

where $f_{\alpha}^{\dagger}$ creates a quasiparticle in orbital $\alpha$. For the dimer, we have $\alpha=\{ \pm, \sigma\}$ where \pm designates the even/odd orbital, $\sigma=\uparrow, \downarrow$ is the spin index and $f_{ \pm \sigma}=$ $\frac{1}{\sqrt{2}}\left(f_{1 \sigma} \pm f_{2 \sigma}\right)$. The molecular eigenstates of the physical Hilbert space of the dimer have the following representation, $\stackrel{41}{ }$ which satisfy the constraints (13)

$$
|\underline{\Gamma}\rangle \equiv \frac{1}{\sqrt{D_{\Gamma}}} \sum_{\Gamma^{\prime}}^{\prime} \phi_{\Gamma \Gamma^{\prime}}^{\dagger}|\mathrm{vac}\rangle\left|\Gamma^{\prime}\right\rangle
$$

where $D_{\Gamma}$ is a normalization factor and the primed sum is over states $\left|\Gamma^{\prime}\right\rangle$ which have the same quantum numbers as $|\Gamma\rangle$. The physical electron operators can be expressed at saddle point as a linear combination of the quasiparticle operators $f$ as

$$
d_{\alpha}^{\dagger} \rightarrow \underline{d}_{\alpha}^{\dagger}=R_{\alpha \beta}^{*} f_{\beta}^{\dagger}
$$

where $R_{\alpha \beta}$ is given by

$$
R_{\alpha \beta}=\sum_{\Gamma_{1} \Gamma_{2} \Gamma_{1}^{\prime} \Gamma_{2}^{\prime}} \sum_{\gamma} M_{\gamma \beta}\left\langle\Gamma_{1}\left|d_{\alpha}\right| \Gamma_{2}\right\rangle\left\langle\Gamma_{1}^{\prime}\left|f_{\gamma}\right| \Gamma_{2}^{\prime}\right\rangle \phi_{\Gamma_{1} \Gamma_{1}^{\prime}}^{\dagger} \phi_{\Gamma_{2} \Gamma_{2}^{\prime}} .
$$


In this expression, $M_{\gamma \beta}$ is a matrix of normalization factors which insure that the exact non-interacting solution is recovered in the saddle-point approximation when $U=0$. Its explicit expression in terms of the slave-boson amplitudes can be found in Ref. 41 .

Writing the dimer Hamiltonian as

$$
\underline{H}_{\text {dimer }}=\sum_{\Gamma} E_{\Gamma} \sum_{\Gamma^{\prime}} \phi_{\Gamma \Gamma^{\prime}}^{\dagger} \phi_{\Gamma \Gamma^{\prime}}
$$

we obtain the partition function as a functional integral over coherent Bose and Fermi fields. The constraints of Eq. (13) are enforced including time-independent Lagrange multipliers $\lambda_{0}$ and $\Lambda_{\alpha \beta}$. The fermionic fields can be integrated out and the resulting bosonic action is treated in the saddle-point approximation. The free energy is finally obtained as

$$
\begin{aligned}
\Omega & =-\frac{1}{\beta} \sum_{i \omega_{n}} \operatorname{Tr} \ln \left[-\mathbf{G}_{f}^{-1}\left(i \omega_{n}\right)\right]-\lambda_{0}+ \\
& +\sum_{\Gamma_{1} \Gamma_{2} \Gamma_{1}^{\prime} \Gamma_{2}^{\prime}} \varphi_{\Gamma_{1} \Gamma_{2}^{\prime}}^{*}\left\{\delta_{\Gamma_{1}^{\prime} \Gamma_{2}^{\prime}} \delta_{\Gamma_{1} \Gamma_{2}}\left(\lambda_{0}+E_{\Gamma_{1}}\right)-\right. \\
& \left.-\delta_{\Gamma_{1} \Gamma_{2}} \sum_{\alpha \beta}\left\langle\Gamma_{1}^{\prime}\left|f_{\alpha}^{\dagger} \Lambda_{\alpha \beta} f_{\beta}\right| \Gamma_{2}^{\prime}\right\rangle\right\} \varphi_{\Gamma_{2} \Gamma_{1}^{\prime}} .
\end{aligned}
$$

In this expression, $\varphi_{\Gamma \Gamma^{\prime}} \equiv\left\langle\phi_{\Gamma \Gamma^{\prime}}\right\rangle$ are saddle-point (cnumbers) expectation values of the boson fields and $G_{f}$ is the quasiparticle (auxiliary fermion) Green's function of the impurity model, given by

$$
\mathbf{G}_{f}^{-1}\left(i \omega_{n}\right)=i \omega_{n} \mathbf{1}-\boldsymbol{\Lambda}-\mathbf{R}^{\dagger}\left(\begin{array}{cc}
\Delta_{+}\left(i \omega_{n}\right) & 0 \\
0 & \Delta_{-}\left(i \omega_{n}\right)
\end{array}\right) \mathbf{R},
$$

with $\Delta_{ \pm}$the hybridization function of the even (resp. odd) orbital. The saddle-point approximation is obtained by extremalizing $\Omega$ over the boson amplitudes $\varphi_{\Gamma \Gamma^{\prime}}$ and the Lagrange multipliers $\lambda_{0}, \Lambda_{\alpha \beta}$.

Within this approximation, the self-energy for the physical electron operators consists simply in a constant term and a term linear in frequency, which are orbitaldependent and $\operatorname{read}^{41}$

$$
\Sigma_{d}\left(i \omega_{n}\right)=i \omega_{n}\left(\mathbf{1}-\left[\mathbf{R} \mathbf{R}^{\dagger}\right]^{-1}\right)+\left[\mathbf{R}^{\dagger}\right]^{-1} \mathbf{\Lambda} \mathbf{R}^{-1}-\varepsilon_{0} \mathbf{1},
$$

so that the matrix of quasiparticle weights reads

$$
\mathbf{Z}=\mathbf{R R}^{\dagger}=\left(\begin{array}{cc}
Z_{+} & 0 \\
0 & Z_{-}
\end{array}\right) .
$$

Of course, as mentioned above, this form of the selfenergy should be understood as a low-energy approximation retaining only the constant terms (which renormalize the level position associated with the even- and oddorbitals) and the quasiparticle weights. Lifetime effects, as well as higher order terms in frequency are neglected within the SB mean-field approximation. Accordingly, the physical electron Green's function

$$
\mathbf{G}_{d}=\mathbf{R ~ G}_{f} \mathbf{R}^{\dagger}
$$

retains only the quasiparticle contribution to the spectral functions (note in particular that these spectral functions do not satisfy the normalization sum rule, and instead have spectral weights $Z_{ \pm}$corresponding to the quasiparticle contributions).

From the expression of the intra-dimer energy at the mean-field level

$$
\left\langle H_{\text {dimer }}\right\rangle=\sum_{\Gamma} E_{\Gamma} \sum_{\Gamma^{\prime}}\left|\varphi_{\Gamma \Gamma^{\prime}}\right|^{2},
$$

we see that the quantity

$$
p_{\Gamma}^{\mathrm{SB}} \equiv \sum_{\Gamma^{\prime}}\left|\varphi_{\Gamma \Gamma^{\prime}}\right|^{2}
$$

can be interpreted as the statistical weight associated with the contribution to the low-energy physics of the multiplet state $\Gamma$. Because of the constraint (13) on the slave bosons, these weights are normalized according to $\sum_{\Gamma} p_{\Gamma}^{\mathrm{SB}}=1$. As discussed later in this article, they can be directly compared to the statistical weights computed within the CTQMC algorithm.

\section{ORBITAL-SELECTIVE MOTT TRANSITION IN MOMENTUM SPACE}

\section{A. The different regimes of doping and the transition}

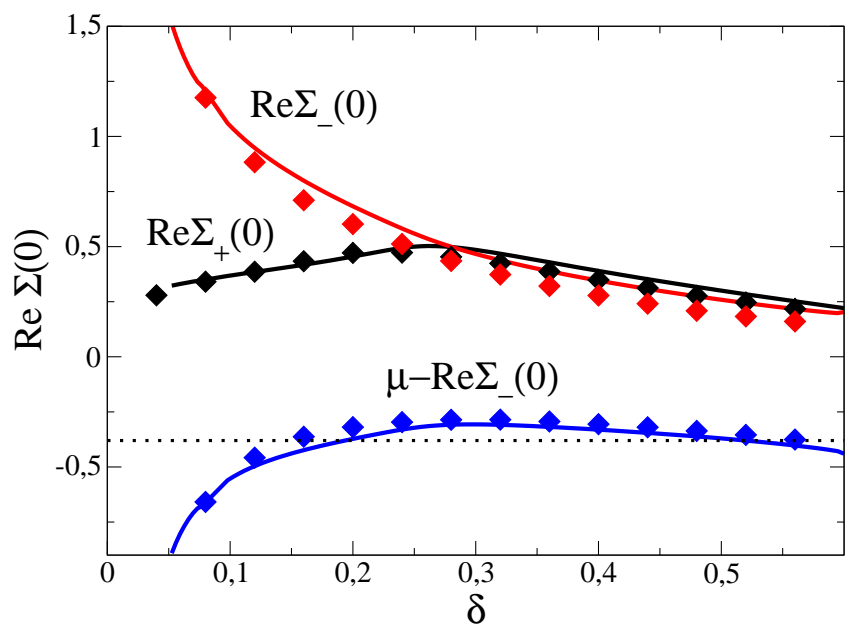

FIG. 3: (Color online) Real part of the even and odd selfenergies at $\omega=0$, extrapolated from CTQMC results for $\beta=200, U=2.5$. The solid lines are the slave-boson (RISB) solution, while the symbols are the CTQMC results. The dotted line is the lower band edge of the $P_{-}$patch represented in Fig. 2

In this section, we describe the behavior of the system in the different regimes of doping. First, we focus on a low-energy analysis which yields a very simple description of the orbital-selective transition. To this end, we 
first analyze the behavior of the real part of the evenand odd-orbital self-energies, extrapolated to zero frequency. This is shown in Fig. 3 using both CTQMC and RISB. Even though the full frequency dependence of the self-energy obtained by CTQMC is highly non-trivial (see subsequent sections), its zero-frequency limit is in remarkable agreement with the RISB solution, as is clear from Fig. 3 .

At large doping $\delta \gtrsim 20 \%, \Sigma_{+}^{\prime}(0)$ and $\Sigma_{-}^{\prime}(0)$ are very close to each other: Both orbitals behave in a similar way. In this large doping regime, the system is a good metal with well-defined quasiparticles everywhere on the Fermi surface. A single-site DMFT description is quite accurate in this regime, since there is little orbital differentiation and hence little momentum dependence.

As the doping level is further reduced $(\delta \lesssim 20 \%)$, the two orbitals start to behave differently, signaling the onset of momentum differentiation in the lattice model. The odd-orbital self-energy $\Sigma_{-}^{\prime}(0)$ increases rapidly as the doping level is reduced, while $\Sigma_{+}^{\prime}(0)$ remains much smaller and even decreases slightly with doping. At the critical doping level $\delta \simeq 16 \%$, the effective chemical potential of the odd orbital $\mu-\Sigma_{-}^{\prime}(0)$ reaches the lower edge $\epsilon_{\min }=-0.38$ of the non-interacting partial density of states corresponding to the outer patch (Fig. 2), as signaled by the dashed horizontal line in Fig. 3. Retaining only the real part of the self-energy, this implies that the pole equation corresponding to the outer patch $\omega+\mu-\varepsilon_{\mathbf{k}}-\Sigma_{-}^{\prime}(\omega)=0$ no longer has solutions for $\omega=0$, signaling the disappearance of low-energy quasiparticle excitations from the outer patch. Hence, for $\delta \lesssim 16 \%$, we have a strongly momentum-differentiated metal, with quasiparticles present only within the central patch. Only when the doping eventually reaches $\delta=0$ do these quasiparticles in turn disappear, corresponding to a Mott insulator. Note that in the above analysis we neglected the contribution from the imaginary part of the self-energy $\Sigma_{-}^{\prime \prime}(0)$. Indeed, our data indicates that $\Sigma_{-}^{\prime \prime}(0)$ vanishes as the temperature goes to zero, so that the transition does exist in this limit. At finite temperatures, the imaginary part of the self-energy gives a small contribution to the spectral density at the chemical potential, but still a rapid change of behavior is expected around the transition.

Further insights into this transition can be obtained by analyzing the average occupancies in each orbital $n_{+}$ and $n_{-}$obtained by CTQMC and within the RISB calculation, see Fig. 4 At large doping, the occupancies obtained by both methods behave similarly and increase with decreasing doping. As the doping gets closer to the critical value $\delta \simeq 16 \%$, the RISB solution displays a strong deviation where $n_{+}^{\text {RISB }}$ increases rapidly and $n_{-}^{\text {RISB }}$ vanishes. Recalling that the slave-boson approximation only accounts for the low-energy physics associated with quasiparticles, this indicates that the odd orbital becomes empty at low energy in the low-doping phase, and that there are no low-energy excitations left in the outer patch, as discussed above. A change of behavior in $n_{ \pm}$

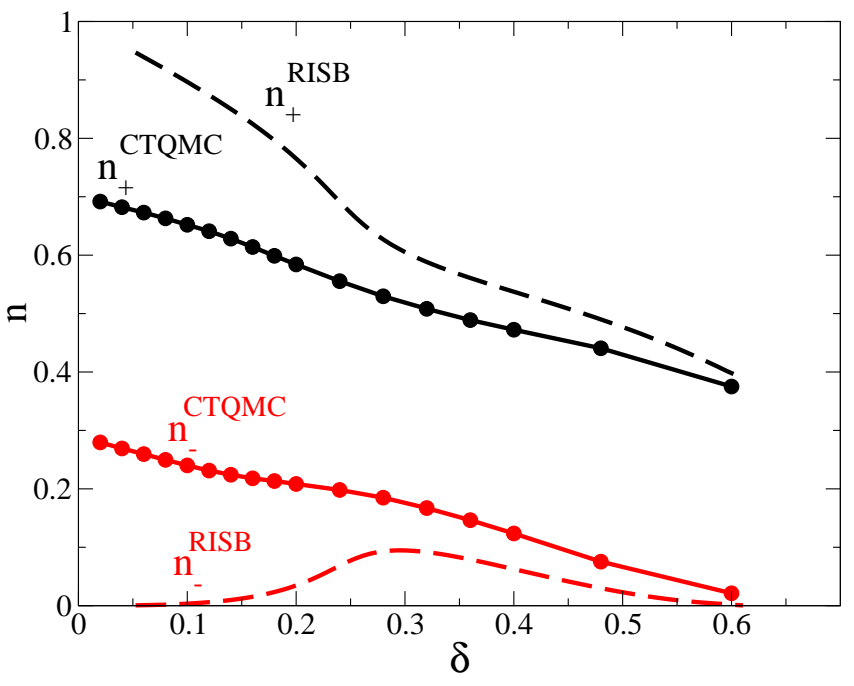

FIG. 4: (Color online) Averages occupancies of the even and odd orbitals, obtained with RISB method (dashed lines) and CTQMC (solid lines with circles). $\beta=200, U=2.5$.

at the transition is also present in the CTQMC solution, but it should be kept in mind that these quantities then include contributions from the higher-energy features of the spectral function, and hence $n_{-}^{\mathrm{CTQMC}}$ is not expected to vanish in the low-doping phase because the odd orbital does have spectral weight at sufficiently negative energies in that phase as well.

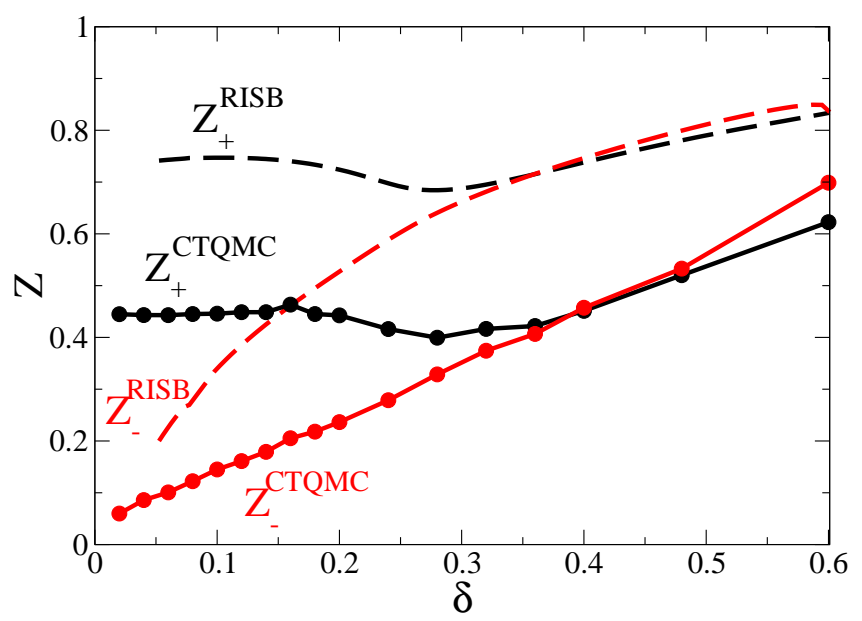

FIG. 5: (Color online) Quasiparticle residues of the even and odd orbitals obtained with RISB method (dashed lines) and CTQMC (solid lines with circles). $\beta=200, U=2.5$.

In our VB-DMFT approach, the strong differentiation in momentum space at low doping manifests itself as an orbital-selective transition: As one approaches the Mott insulator, the odd orbital localizes at a finite doping level, while the even one only does so at $\delta=0$ when reaching the Mott insulator. This is actually a crude description of the formation of Fermi arcs. Indeed, for $\delta<16 \%$, quasiparticles are only present in the inner patch, close to the 
nodal region. Instead, in the antinodal region, the Fermi surface is destroyed and the spectral function vanishes at the chemical potential. A more precise description of the actual formation of the Fermi arcs requires to specify a procedure for reconstructing the momentum dependence of the self-energy from this two-orbital description: This is the topic of Sec. $\mathrm{V}$.

A marked difference of behavior between the two orbitals at low doping is also found for the quasiparticle residues (see Fig. 5) defined by

$$
Z_{ \pm}=\left(1-\left.\frac{d \Sigma_{ \pm}^{\prime}(\omega)}{d \omega}\right|_{\omega \rightarrow 0}\right)^{-1}
$$

The CTQMC data and RISB approximation for $Z_{ \pm}$differ in absolute value but they both display similar trends. Again, at high doping $Z_{+}$and $Z_{-}$are close to each other. As the doping is reduced, $Z_{-}$decreases (with roughly a linear dependence on doping) while $Z_{+}$remains essentially constant. Below the critical doping, $Z_{-}$cannot be interpreted as the spectral weight of a quasiparticle (the odd orbital is localized), but it does indicates that the correlations continue to affect the odd-orbital self-energy. Hence, correlations preferentially act on the antinodal electrons. In contrast, correlations appear to have little influence on $Z_{+}$below $\delta_{c}$, indicating that the nodal quasiparticles appear to be "protected" by the opening of the (pseudo-) gap in the antinodal regions.

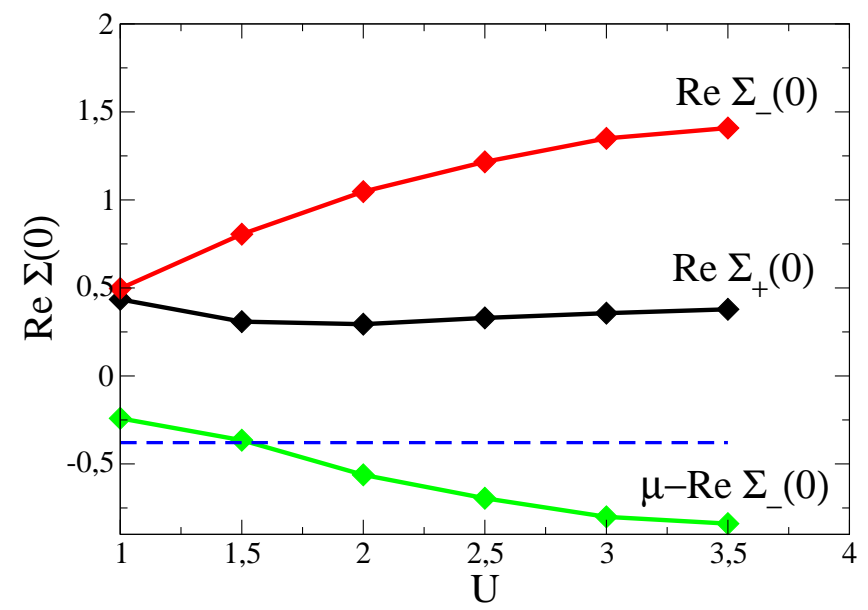

FIG. 6: (Color online) Real parts of the self-energies at $\omega=0$, extrapolated from CTQMC results at $\beta=200$, as a function of $U$ at a fixed doping $\delta=8 \%$. The dotted line is the lower band edge of the $P_{-}$patch represented in Fig. 2

The value of the critical doping $\delta_{\mathrm{c}}$ at which the transition appears depends on the value of the interaction $U$. The larger $U$, the larger $\delta_{\mathrm{c}}$. To illustrate the effect of $U$, we plot, in Fig. 6, the real parts of the self-energies extrapolated to zero frequency for different values of $U$ at a fixed doping $\delta=8 \%$. The difference between the even and the odd orbital increases with $U$. Above $U \simeq 1.5$, the renormalized chemical potential falls below the lower edge of the partial DOS for the outer patch and the odd spectral function is vanishing at the chemical potential. However, when $U<1.5$, the odd orbital is metallic again, showing clearly that the Coulomb interaction is at the origin of the differentiation in momentum space.

\section{B. Spectral functions and the pseudogap at low doping}

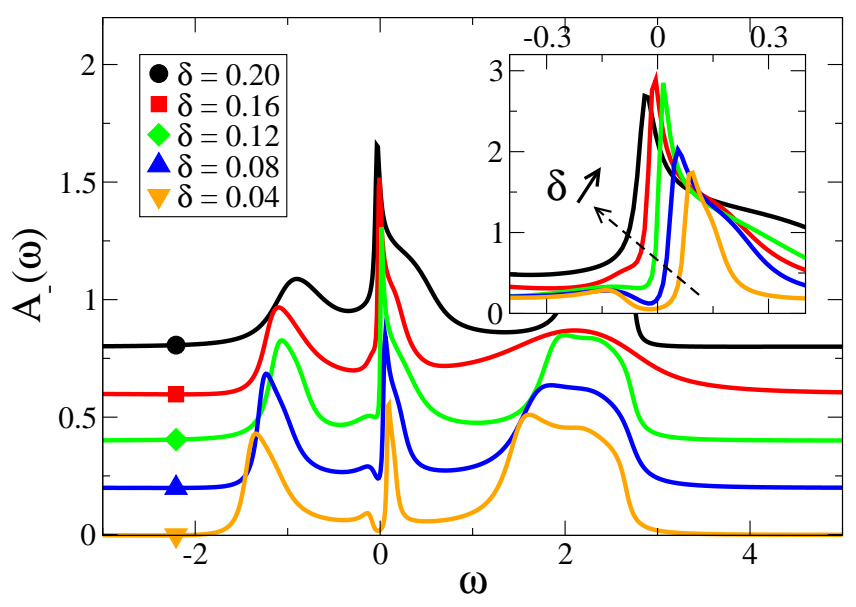

FIG. 7: (Color online) Spectral function $A_{-}(\omega)$ for the odd orbital, obtained with Padé approximants (see Appendix B), for various dopings at $\beta=200$. A shift of 0.3 has been added between the curves for clarity. Inset: Zoom of the same curves at low frequencies (no shift added).

In the previous section, we have shown that strong orbital differentiation sets in at low-doping levels $\delta \lesssim$ $16 \%$. In a simplified low-energy description, the effective chemical potential for the odd orbital is pushed below the lower band edge. This corresponds to the vanishing of the low-energy spectral weight of the odd orbital, and signals the disappearance of low-energy quasiparticles in the antinodal regions. In this section, we go beyond this simple low-energy analysis and study the full frequency dependence of the spectral functions of both the even and odd orbitals. One of the main outcomes of this study, as we shall see, is that the odd orbital does not have zero spectral weight in a finite frequency range around $\omega=0$, but rather develops a pseudogap.

The computation of real-frequency spectral functions is made possible by the very high quality of the CTQMC results on the Matsubara axis, allowing for reliable analytical continuations to the real axis at low and intermediate energy, using simple Padé approximants 42 (see Appendix B . This is a definite advantage of our simplified two-orbital approach, in which the statistical noise of Monte Carlo data can be reduced down to very small values at a reasonable computational cost. In Fig. 7, we plot the spectral function $A_{-}(\omega)$ of the odd orbital at a fixed interaction $U=2.5$. At high energies, the spectra display the expected lower and upper Hubbard bands, and from now we focus on the lower energy range. In 
this range, the spectra display a central peak. At high doping, this peak is centered at the Fermi level $\omega=0$. As the doping level is reduced, this peak shifts toward positive energies. At the critical doping $\delta_{c} \simeq 16 \%$, the chemical potential is at the lower edge of the peak, in agreement with the low-energy analysis discussed above.

Correspondingly, the spectral weight at $\omega=0$ is strongly suppressed as the doping is reduced from $\delta_{c} \simeq$ $16 \%$. A pseudogap is formed at low energy, as clear from the inset of Fig. 7, which deepens as the doping level is reduced. There is no coherent spectral weight at the chemical potential. The finite spectral weight at $\omega=0$ is due to thermal excitations. In contrast, the finite spectral weight at small but non-zero frequency survives as temperature is reduced, corresponding to a pseudogap rather than a true gap in $A_{-}(\omega)$.

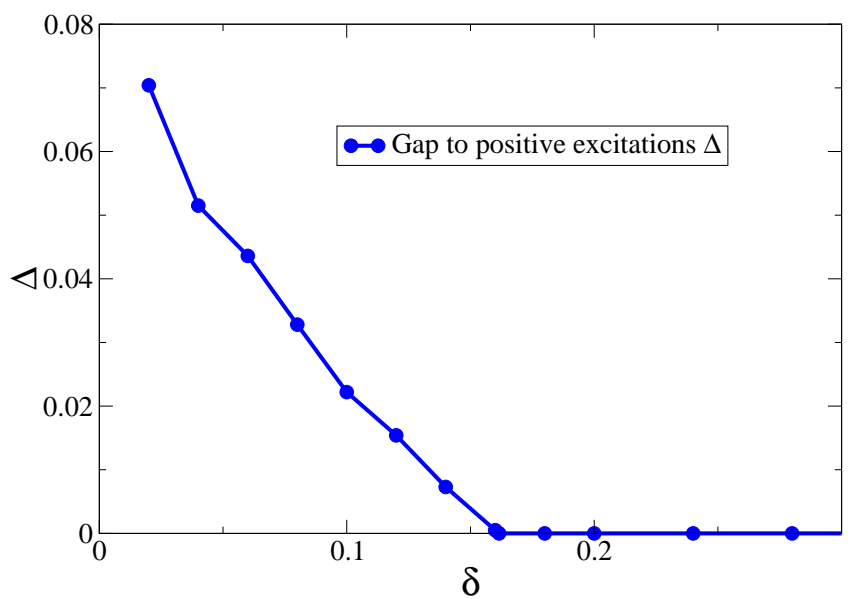

FIG. 8: (Color online) Gap to positive coherent excitations in the odd orbital, obtained from Eq. (28).

The prominent peak at low energies in $A_{-}(\omega)$ is associated with the first coherent excitations at positive energies. By neglecting the effect of the imaginary part of the self-energy, it is possible to precisely identify the position of this peak as the scale $\Delta$ where the first positive-energy poles appear in the expression of the odd-orbital Green's function

$$
G_{-}(\omega)=\sum_{\mathbf{k} \in \mathcal{P}_{-}} \frac{1}{\omega+\mu-\varepsilon_{\mathbf{k}}-\Sigma_{-}^{\prime}(\omega)} .
$$

Hence, $\Delta$ is the solution of

$$
\Delta+\mu-\epsilon_{\min }-\Sigma_{-}^{\prime}(\Delta)=0,
$$

where $\epsilon_{\min }$ is the lower-band edge of the outer-patch partial DOS. The solution of this equation is shown in Fig. 8 , The gap $\Delta$ opens below $\delta_{c}$ and provides a characteristic energy scale for the position of the peak, see inset in Fig. 7 Note that this energy scale is much smaller than the deviation of the renormalized chemical potential $\mu-\Sigma_{-}^{\prime}(0)$ from the lower outer-patch band edge because of the non-trivial frequency behavior of the self-energy.
Furthermore, the magnitude of $\Delta$ as obtained from Fig. 8 is in the range of tens of meV's consistent with the typical magnitude of the pseudogap in cuprates.

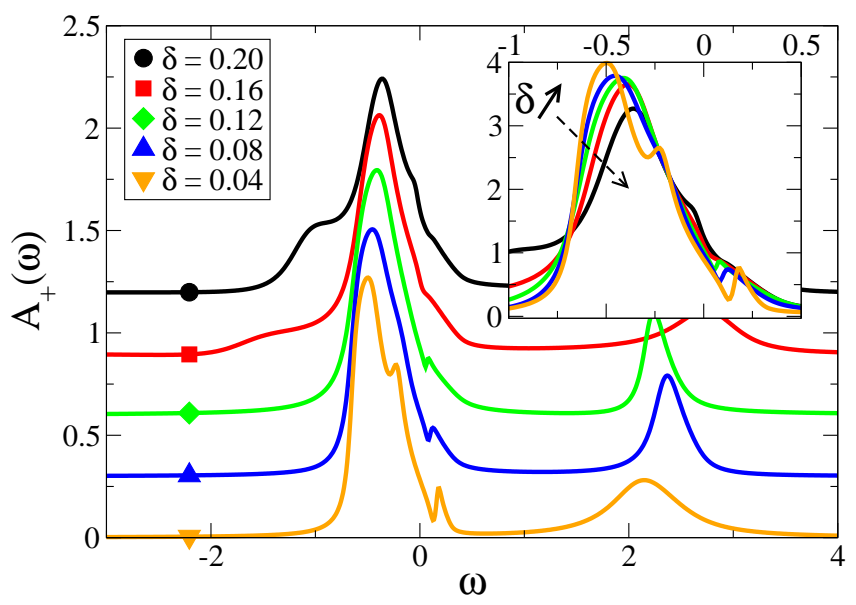

FIG. 9: (Color online) Spectral function $A_{+}(\omega)$ for the even orbital, obtained with Padé approximants (see Appendix B), for various dopings at $\beta=200$. A shift of 0.2 has been added between each curves for clarity. Inset: Zoom of the same curves at low frequencies (no shift added).

In Fig. 9, we display the spectral function $A_{+}(\omega)$ of the even orbital for different doping levels. The dependence of $A_{+}(\omega)$ on doping is rather weak. The main feature of the non-interacting density of states corresponding to the central patch (Fig. 2) is recovered on these spectra, namely a broad peak centered at negative energy with a tail leaking above the Fermi level. The absence of a visible lower Hubbard band, as well as the relatively small spectral weight of the upper Hubbard band (at the same position $\omega \simeq 2$ as in $A_{-}(\omega)$ ), indicate that correlations have a much weaker effect on the even orbital (central patch, nodal regions) than on the odd (antinodal) one, as already anticipated in the previous section. The spectral function $A_{+}(\omega)$ is quite asymmetric, with more hole-like excitations than particle-like excitations (in line with the fact that the central patch corresponds mainly to filled states). At low doping, a small dip appears close to the chemical potential. The position of this dip is close to that of the prominent peak in the odd-orbital spectral function.

\section{Comparison with tunneling experiments}

A direct comparison can be made between our VBDMFT cluster calculations and tunneling experiments in the normal state of cuprate superconductors. Indeed, tunneling directly probes the momentum-integrated spectral density, and hence the comparison is free of the possible ambiguities associated with momentum-space reconstruction which influence the comparison of cluster calculations to momentum-resolved spectroscopies (as 


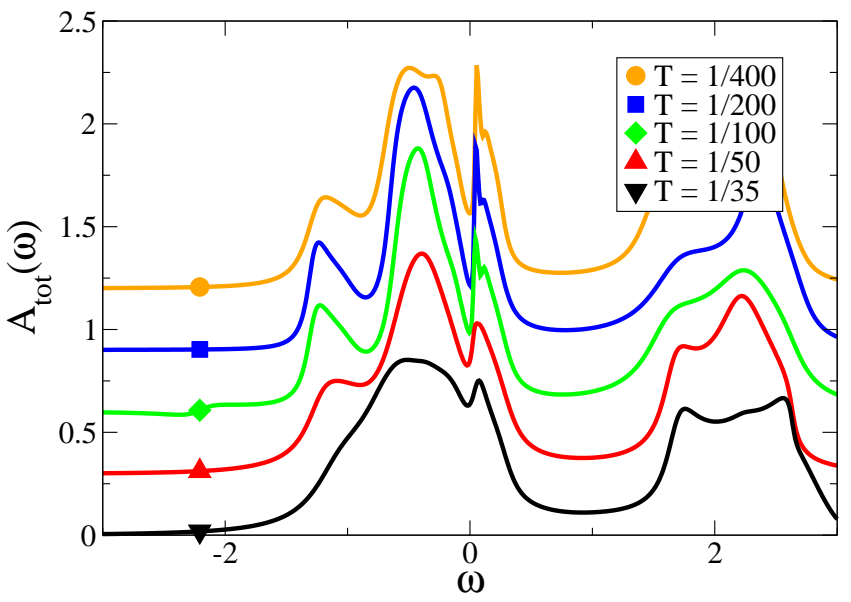

FIG. 10: (Color online) Total spectral function $A_{\text {tot }}(\omega)$ for various temperature at $\delta=0.08$. A shift of 0.3 has been added between each curves for clarity.

discussed in more detail in Sec. V] . The tunneling conductance $d I / d V$ as a function of the voltage $V$ is given by $\underline{48}$

$$
\frac{d I}{d V} \propto \int_{-\infty}^{+\infty} d \omega\left[-f^{\prime}(\omega-e V)\right] A_{\mathrm{tot}}(\omega)
$$

In this expression, tunneling between a normal metal (with a featureless density of states) and the correlated sample is considered, $f^{\prime}$ designates the derivative of the Fermi function, $e>0$ is the absolute value of the electron charge and $A_{\text {tot }}(\omega)$ is the local (momentum-integrated) spectral function. The energy dependence of tunneling matrix elements has been neglected, and the correlated sample is considered to be homogeneous.

In Fig. 10, we display $A_{\text {tot }}(\omega)=A_{+}(\omega)+A_{-}(\omega)$ for different temperatures $T=1 / \beta$ at a fixed doping $\delta=8 \%$. In this local spectral function, we recognize the features discussed above, namely the broad band at negative energy originating from $A_{+}(\omega)$, and the sharp peak at a small positive energy found in $A_{-}(\omega)$, separated by the pseudogap at low energy.

In Fig. 11, we display the voltage dependence of the tunneling conductance obtained using the spectral function $A_{\text {tot }}(\omega)$ calculated within VB-DMFT. The results are displayed at a fixed, low-doping level $\delta=8 \%$ in the pseudogap regime, for different temperatures. For comparison, we also display the experimental data of Renner

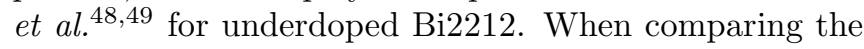
two set of curves, attention should be paid to the fact that our calculation applies at this stage only to the normal state $T>T_{c}$. Our calculation compares quite favorably to the experimental data, in several respects. At low temperature, both the theoretical and experimental conductance displays i) a dip at low voltage corresponding to the pseudogap ii) a peak at a small positive voltage (corresponding to empty hole-like states) and iii) an overall particle-hole asymmetry $d I / d V<0$ at negative voltage as well as at positive voltage above the peak, as indeed expected in a doped Mott insulator. Furthermore, we observe that the temperature dependence reveals the gradual buildup of the positive-voltage coherence peak as temperature is lowered, as well as the gradual opening of the pseudogap at low voltage. One aspect of our theoretical results which departs from the experiments is the detailed shape of the conductance at negative voltage: In experiments a more pronounced dip is visible, while our results rather display a gradual, linear-like decrease.

Our results have direct implications for the interpretation of tunneling experiments, and also suggest some further experiments to test these predictions. First, the coherence peak at small positive voltage must be associated, according to our theory, mainly with low-energy empty states in the antinodal regions. Second, the position of this peak is predicted to have a definite doping dependence, tracking $\Delta$ in Fig. 8 and hence moving to higher energy as the doping level is reduced from 'optimal' doping.

\section{Frequency-dependence of the self-energy and the inelastic scattering rates}

Here, we discuss the frequency dependence of the imaginary part of the self-energies $\Sigma_{ \pm}^{\prime \prime}(\omega) \equiv \operatorname{Im} \Sigma_{ \pm}\left(\omega+\mathrm{i} 0^{+}\right)$ and its physical implications for the inelastic scattering rates of the nodal and antinodal quasiparticles in the different regimes of doping. These quantities are displayed on Figs. 12, 13, Let us recall that these quantities are directly related to the quasiparticle lifetimes, which is given by the inverse of $Z_{ \pm} \Sigma_{ \pm}^{\prime \prime}(\omega)$.

Again, we observe that at large doping, these quantities have rather similar behavior. An approximately quadratic frequency dependence is found at low energy, corresponding to a Fermi liquid behavior of both orbitals, and the self-energies display high-energy peaks corresponding to the structures in the spectral functions described above. Overall, the self-energies at large doping are quite similar to those found in the single-site DMFT description of a correlated Fermi liquid.

The situation becomes radically different as the doping level is reduced. The first observation is that the overall scale for $\Sigma_{+}^{\prime \prime}(\omega)$ and for $\Sigma_{-}^{\prime \prime}(\omega)$ then becomes very different. Clearly, away from the very low-energy region, $\Sigma_{-}^{\prime \prime}(\omega)$ becomes much larger than $\Sigma_{+}^{\prime \prime}(\omega)$, indicating again a stronger effect of correlations on the antinodal regions (odd orbital) than on the nodal ones (even orbital), and a much larger degree of coherence of the nodal quasiparticles.

Focusing on the even orbital (nodes) at low frequency, we observe that $\Sigma_{+}^{\prime \prime}(\omega=0)$ displays a marked decrease as the doping level is reduced from the characteristic doping $\delta_{c} \simeq 16 \%$ at which orbital differentiation sets in and the pseudogap opens. Physically, this means that the opening of the pseudogap leads to a protection of the nodal quasiparticles by increasing their inelastic lifetime at low 

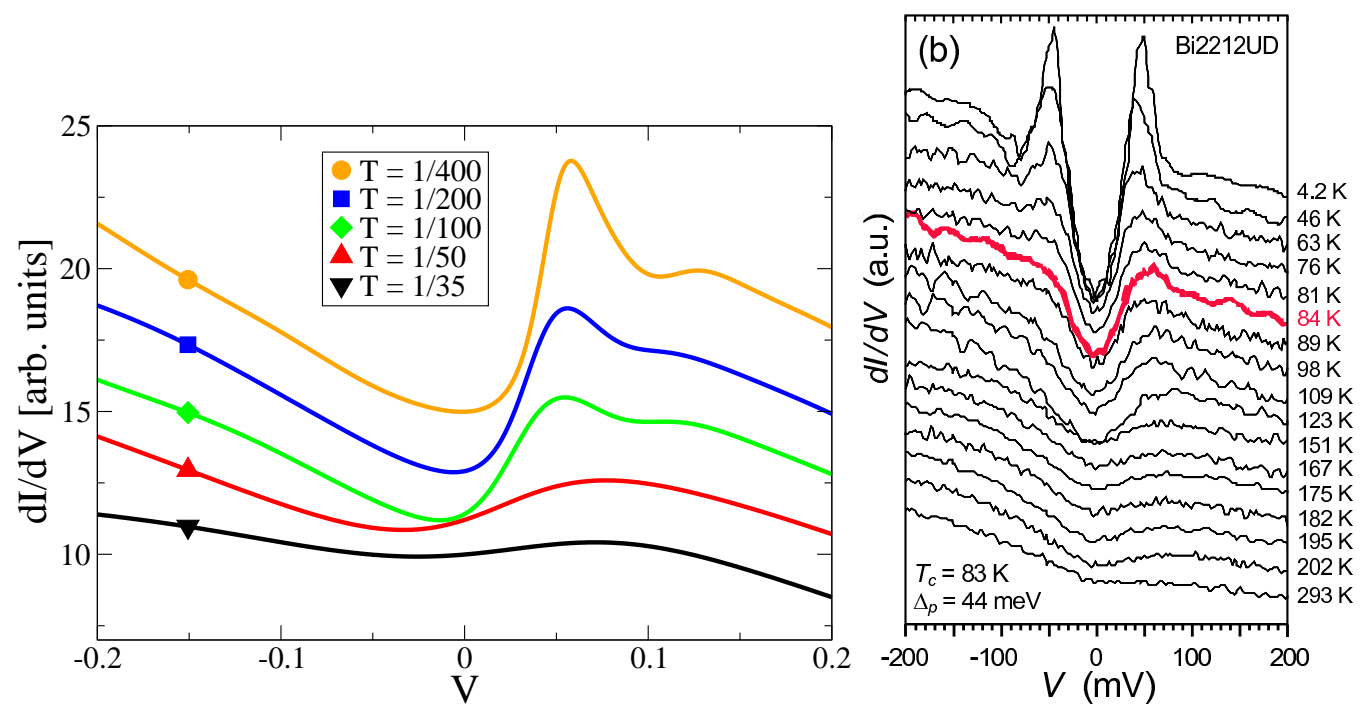

FIG. 11: (Color online) Left panel: STM curve for various temperature at $\delta=0.08$, in arbitrary units. A shift has been added between each curves for clarity. Right panel: DOS measured by STM experiments on Bi2212 with $T_{c}=83 K$. Figure reprinted with permission from Ref. 48 (Fig. 22b). Copyright 2007 by the American Physical Society.
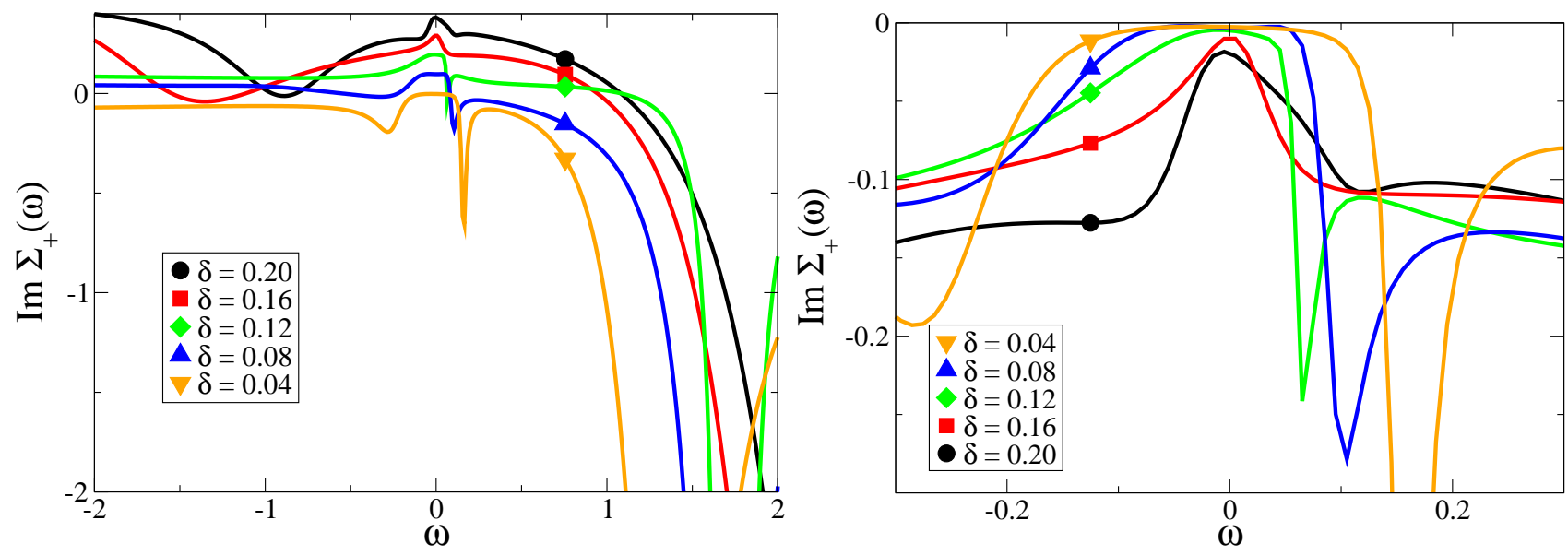

FIG. 12: (Color online) Left panel: Imaginary part of the self-energy $\Sigma_{+}(\omega)$ for the even orbital. A shift of 0.1 has been added between the curves for clarity. Right panel: Zoom over the low-frequency region (no shift added).

energy. Indeed, $\Sigma_{+}^{\prime \prime}(\omega)$ displays a quite remarkable shape at low doping, with a rather large interval of frequency around $\omega=0$ in which it is very small and flat, indicating almost free nodal quasiparticles at low doping.

This is in marked contrast to the behavior of the odd (antinodal) orbital. In this case, our real-frequency data lack the precision required to assess precisely the doping dependence of the very low-frequency rate $\Sigma_{-}^{\prime \prime}(\omega=0)$. However, as soon as one focuses on a small but finite frequency (which indeed is relevant to the lifetime of antinodal quasiparticles at the edge of the pseudogap), it is apparent from Fig. 13 that $\Sigma_{-}^{\prime \prime}(\omega)$ rapidly increases as the doping is reduced from $\delta_{c}$. This corresponds to increasingly incoherent antinodal quasiparticles at low doping level.

Making contact with experiments, these observations appear to be in good qualitative agreement with the fact that the in-plane resistivity of cuprate superconductors is reduced when the pseudogap opens and that nodal quasiparticles survive at low doping while the antinodal ones loose their coherence.

\section{E. Other patches}

So far we have presented results for a particular patching scheme of the BZ. The motivation behind this choice is based on the known phenomenology of the cuprates: The central patch is shaped in such a way as to contain the nodal point while the outer patch is modeled to contain the incoherent antinodal region. Phenomenological patch models using related patches have been used to 

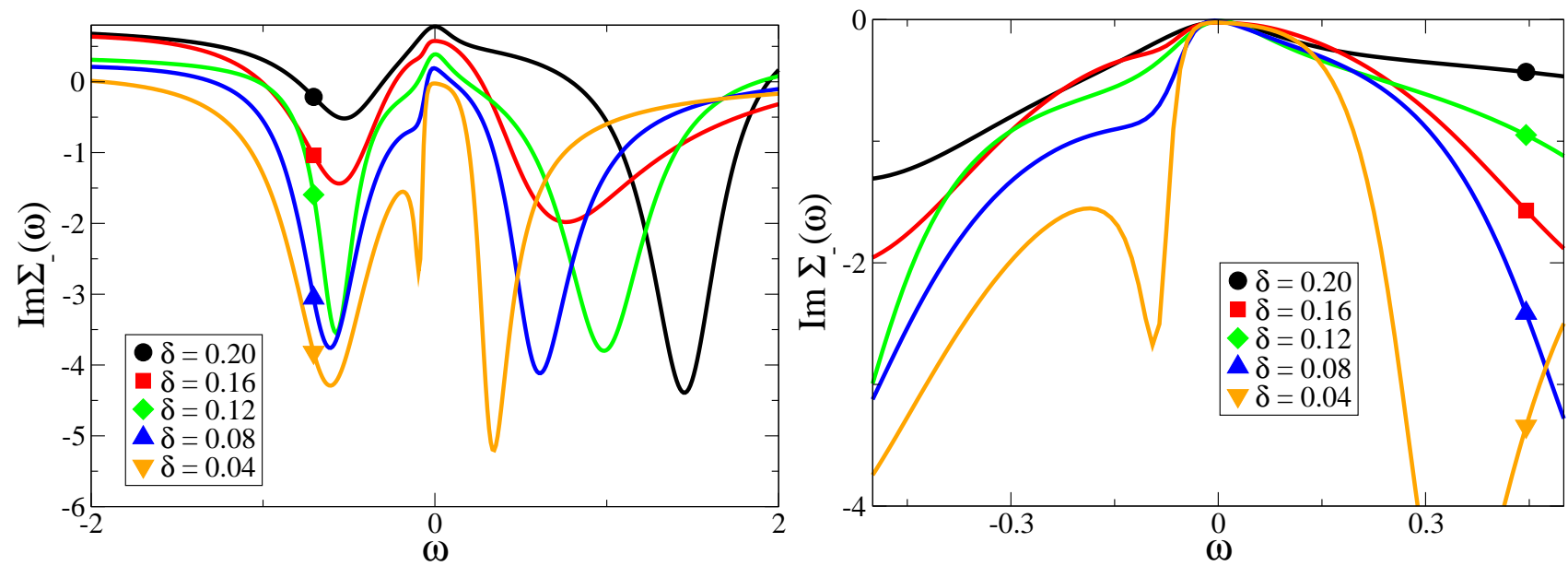

FIG. 13: (Color online) Left panel: Imaginary part of the self-energy $\Sigma_{-}(\omega)$ for the odd orbital. A shift of 0.2 has been added between the curves for clarity. Right panel: Zoom over the low-frequency region (no shift added).

parametrize the transport properties of the cuprate superconductors,$\frac{50}{}$ As we saw, reducing the doping induces a transition in which the outer patch becomes insulating. Here we address the stability of this picture with respect to the deformation of the patches. We consider patches that do not break the lattice point symmetry and have equal volume not to incur into problems with the definition of the cluster Hamiltonian (for details on the formalism see Appendix A).

The main difference between the patching schemes that we consider (see Fig. 14d) is the relative weight given to the nodal and antinodal regions. Compared to the reference patching used in the rest of the paper (patching $\mathrm{B}$, also in Fig. 10, in patching $\mathrm{A}$ the central patch includes the node and also large part of the antinode. At the opposite, in patching $\mathrm{C}$ the outer patch has a larger contribution of the node.

In Fig. 14a-c we present results for the three patching schemes of Fig. 14 $\mathrm{d}$. The results for the self-energy and the occupations are qualitatively very similar for the different patching schemes. Most importantly, the distinctive feature of a selective insulating transition as the doping is decreased remains in all the cases. An analysis of $\mu-\operatorname{Re} \Sigma_{-}(0)$ allows us to calculate the critical doping as indicated by the arrows in Fig. 14k.

We notice how, by increasing the portion of the nodal region contained in the outer patch, the critical doping shifts systematically to lower values. This trend is consistent with a picture in which the quasiparticles are restricted to a fraction (an arc) of the Fermi surface located near the node. By decreasing the doping the quasiparticles disappear at the sides of the arc inducing the selective transition in the outer orbital. Despite the similarity of the trend with the experimental behavior in cuprates, the sensitivity of the method is not sufficient to obtain a quantitative prediction for the size of the arc as a function of doping.

We also investigated the real-space cluster method
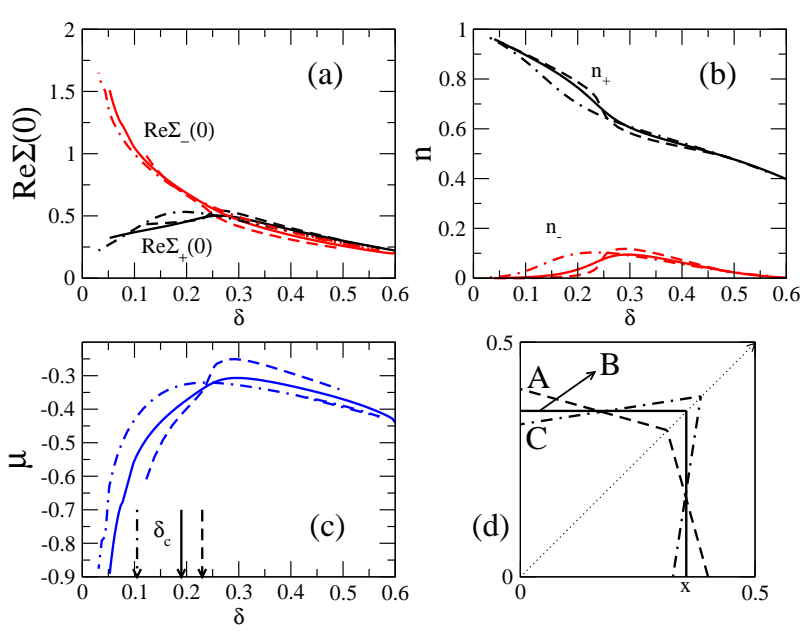

FIG. 14: Doping dependence of (a) the real part of $\Sigma_{ \pm}(0),($ b) the even and odd orbital occupations, and (c) $\mu-\Sigma_{-}(0)$. The arrows in (c) indicate the critical doping where the odd band becomes insulating. Results for different patching schemes as shown in (d) using the RISB method.

CDMFT (cellular-DMFT) $\stackrel{4,18}{\Perp}$ In this method, the notion of patches in the Brillouin zone cannot be introduced because the starting point is a real-space cluster that breaks translational symmetry. Nevertheless the qualitative picture emerging from CDMFT is very similar to the one found in DCA. In particular, we find also in CDMFT a critical doping below which the system is in a selective Mott-insulating state. 


\section{PHYSICS OF THE EFFECTIVE TWO-IMPURITY MODEL AND THE ROLE OF THE SELF-CONSISTENCY}

In this section, we relate and contrast the orbitalselective transition described above to the physics of the crossover between a Kondo-dominated and a singletdominated regime in the two-impurity Anderson model.

\section{A. Singlet dominance at low doping}

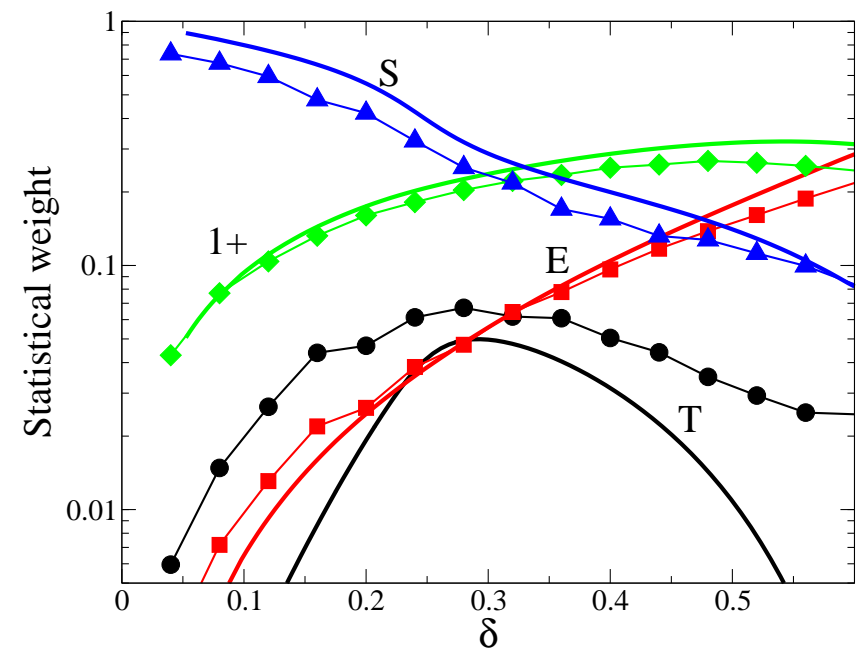

FIG. 15: (Color online) Statistical weights of the various dimer cluster eigenstates (labeled as in Table I). $S$ is the intra-dimer singlet, $1+$ the (spin-degenerate) state with one electron in the even orbital, $E$ the empty state and $T$ the intra-dimer triplet. $\beta=200$.

A way to obtain a more transparent physical picture of the phases encountered as a function of doping is to study the contribution of the different cluster eigenstates to the density matrix. We plot in Fig. 15 the statistical contribution of several cluster eigenstates $|\Gamma\rangle$ (see Sec. IIB) from CTQMC ( $p_{\Gamma}^{\mathrm{QMC}}$ defined in Eq. 11) and the RISB method ( $p_{\Gamma}^{\mathrm{SB}}$ defined in Eq. 25).

The agreement between CTQMC and RISB is very good, and even quantitative for the two states with highest weights. At large doping, the empty state and the two spin-degenerate states with one electron in the even orbital dominate, as expected. As doping decreases, these states lose weight and the intra-dimer singlet prevails, reflecting the strong tendency to valence-bond formation. Therefore, the orbital (momentum) differentiation at low doping is governed by intra-dimer singlet formation. This situation is strongly reminiscent of the behavior of the two-impurity Anderson model (2IAM) $.51,52$ In the following subsection we will compare the results for the 2IAM and VB-DMFT to find the extent of this similarity.

\section{B. Role of the self-consistency: from a RKKY/Kondo crossover to a transition}

The 2IAM has been thoroughly studied by many authors using a variety of methods $51,53,54,55,56$ Here we will focus on a simplified version of the 2IAM where the impurities are coupled directly through a hopping term $\bar{t}$. In standard notation the Hamiltonian is given by

$$
\begin{aligned}
H_{2 I A M} & =U\left(n_{1 \uparrow} n_{1 \downarrow}+n_{2 \uparrow} n_{2 \downarrow}\right)+\varepsilon_{0} \sum_{\sigma \alpha} n_{\alpha \sigma} \\
& -\bar{t} \sum_{\sigma}\left(d_{1 \sigma}^{\dagger} d_{2 \sigma}+H . c .\right)-V \sum_{\mathbf{k} \sigma}\left(d_{1 \sigma}^{\dagger} c_{\mathbf{k} 1 \sigma}+H . c .\right) \\
& -V \sum_{\mathbf{k} \sigma}\left(d_{2 \sigma}^{\dagger} c_{\mathbf{k} 2 \sigma}+H . c .\right)+\sum_{\mathbf{k} \alpha \sigma} \varepsilon_{\mathbf{k}} c_{\mathbf{k} \alpha \sigma}^{\dagger} c_{\mathbf{k} \alpha \sigma},
\end{aligned}
$$

where $U$ is the local repulsion and $\varepsilon_{0}$ the level energy. Note that each impurity is coupled to an independent electronic bath and that there are no crossed baths that would couple to both impurities. We choose the baths to have a semi-elliptic density of states of half-bandwidth $D=1$ and the hybridization $V=0.5 D$. This model corresponds to the impurity model that is solved in VBDMFT, with the important difference that the baths are kept fixed and $\Delta_{12}=0$.

For $\bar{t}=0$ the problem reduces to that of two independent single-impurity Anderson models. The RISB method (as other slave-boson approaches) provides a description of the quasiparticles in the Kondo resonance. The impurity spectral density has a single peak at the Fermi level whose width is of the order of the Kondo energy $T_{K}$. The real part of the self-energy in the electron-hole symmetric situation $\left(\varepsilon_{0}=-U / 2\right)$, is simply $\operatorname{Re} \Sigma_{ \pm}(0)=U / 2$ while $Z$ decreases monotonously with increasing $U$ and exponentially for large $U$ (see Fig. 16). When $\bar{t}$ is turned on, an antiferromagnetic coupling $I=4 \bar{t}^{2} / U$ is generated between the impurities. If $I \lesssim T_{K}$ (see Fig. 16 for $U \lesssim 4$ ) both impurities are in the Kondo regime as in the $\bar{t}=0$ case and the behavior of $\operatorname{Re} \Sigma_{ \pm}(0)$ and $\mathrm{Z}$ reproduces that of uncoupled impurities. In the opposite limit (large $U$ in Fig. 16) there is a large inter-impurity correlation which is signaled by a differentiation between $\operatorname{Re} \Sigma_{+}(0)$ and $\operatorname{Re} \Sigma_{-}(0)$ (i.e. the emergence of a $\operatorname{Re} \Sigma_{12}(0)$ term).

The behavior of the bosonic amplitudes with increasing interaction $U$ clearly shows the two regimes and the crossover region (see Fig.16d). In the Kondo regime both the singlet and triplet multiplets have large amplitudes, while in the so-called RKKY (Ruderman-Kittel-KasuyaYosida) regime the singlet dominates the physics and its associated bosonic amplitude is close to one. Since the level occupation is related to the bosonic amplitudes through the constraints of Eq. (13), their behavior follows.

In the RKKY regime, the even orbital (where the ground state singlet lives) fills up while the odd orbital empties (see Fig. 16) as in earlier studies of $S U(N)$ two-impurity models in the large- $N$ limit. 54 The RISB 

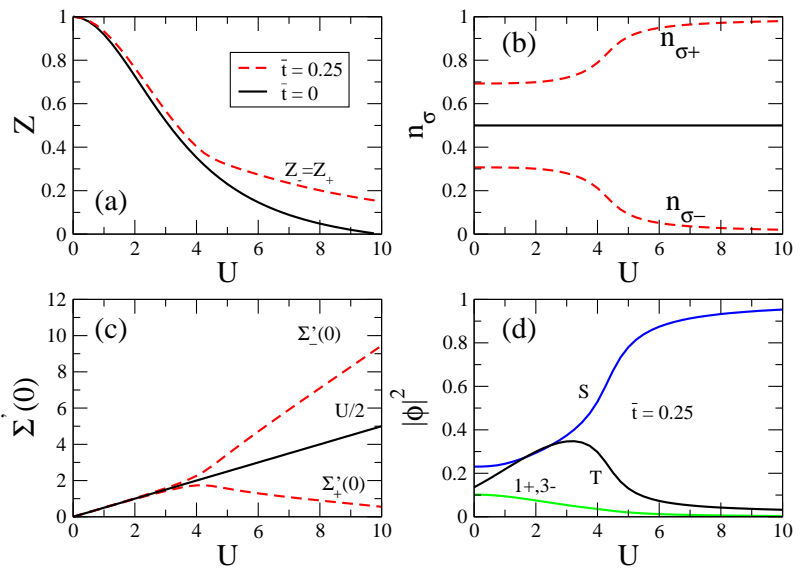

FIG. 16: Kondo to RKKY crossover as a function of the coulomb repulsion $U$ at the impurities at half-filling. (a) Quasiparticle weights. (b) Even and odd orbital occupations. (c) Real part of the self-energy. (d) Boson amplitudes for the intra-dimer singlet $(\mathrm{S})$, the intra-dimer triplet $(\mathrm{T})$, and the spin degenerate doublets with one electron and even symmetry $(1+)$ and three electrons with odd symmetry (3-).

method brings a significant advantage over previous methods since it generates at the mean-field level the RKKY interaction which in previous treatments had to be introduced in an ad-hoc fashion or by treating fluctuations in higher orders in $1 / N \underline{\underline{57}}$

The crossover can also be observed by changing the impurity occupation, i.e. by shifting the local energy $\varepsilon_{0}$. If Fig. 17, we present different physical quantities as a function of the impurity doping level $\delta^{*}=1-\sum_{\alpha, \sigma} n_{\alpha \sigma}$. At zero doping the system is electron-hole symmetric and for the value of $U=7 D$ in the figure it is in the RKKY regime. This is clearly observed in the boson amplitudes, the level occupations and the real part of the self-energy at zero frequency. Increasing the doping increases the charge fluctuations in the impurity and this enhances the Kondo correlations. At a doping level $\delta^{*} \sim 0.1$ there is a crossover to the Kondo regime where the inter-impurity correlation is small. For large values of $\delta^{*}$ the impurities enter an empty orbital regime an the effect of correlations is small.

The behavior observed as a function of doping for the different quantities of the impurity model closely resembles that of VB-DMFT. Note however that the 2IAM presents a crossover between the Kondo and RKKY regimes while in VB-DMFT there is an orbital-selective Mott transition. The origin of the transition can be traced back to the only difference between VB-DMFT and the 2IAM, namely the presence in VB-DMFT of selfconsistently determined baths. Indeed the odd-orbital Green's function for the 2IAM is

$$
G_{-}(\omega)=\frac{1}{\omega+\mu-\Delta_{-}^{\mathrm{hybr}}(\omega)-\Sigma_{-}(\omega)}
$$

where $\Delta_{-}^{\text {hybr }}(\omega)=2(V / D)^{2} \int d \varepsilon \frac{\sqrt{D^{2}-\varepsilon^{2}}}{\omega-\varepsilon}$. The coarsegrained odd-patch Green's function in VB-DMFT is in-
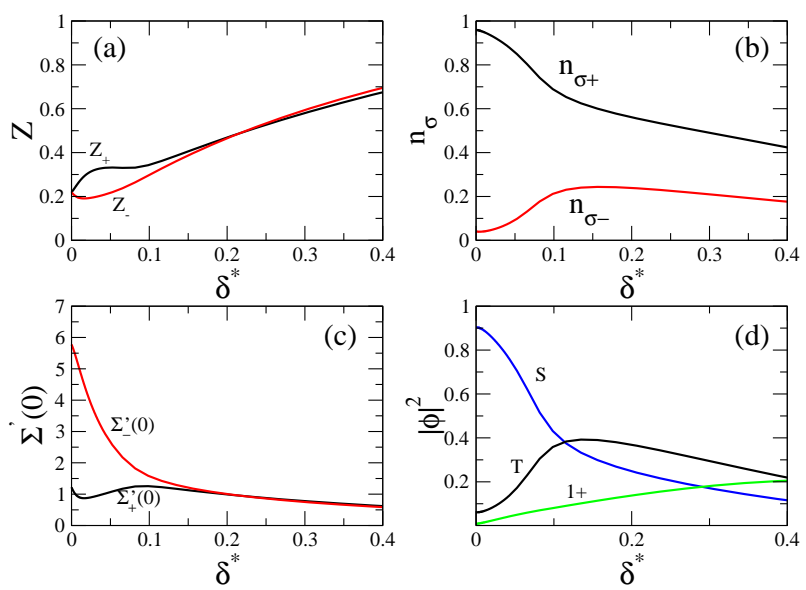

FIG. 17: Kondo to RKKY crossover for the two impurity Anderson model as described by RISB as a function of the impurity level doping $\delta^{*}=1-n$. Parameters are $U=7 D$ and $\bar{t}=0.25 D$. (a) Quasiparticle weights. (b) Even and odd orbital occupations. (c) Real part of the self-energy. B (d) Boson amplitudes for the intra-dimer singlet $(\mathrm{S})$, the intradimer triplet $(\mathrm{T})$, and the spin degenerate doublet with one electron and even symmetry $(1+)$.

stead

$$
G_{-}(\omega)=\sum_{\mathbf{k} \in \mathcal{P}_{-}} \frac{1}{\omega+\mu-\varepsilon_{\mathbf{k}}-\Sigma_{-}(\omega)}
$$

While in the 2IAM the rigid structure of the baths prevents the complete removal of spectral weight from the chemical potential, in VB-DMFT the baths can adjust to allow for such an effect. When performing the VB-DMFT self-consistency loop, $\operatorname{Re} \Sigma_{-}(0)$ acts as a shift of the chemical potential for the odd band. Entering the RKKY regime $\operatorname{Re} \Sigma_{-}(0)$ grows and can become large enough to push the chemical potential off the band and make it insulating. In turn, an insulating odd band enhances the intra-singlet correlations of the dimer making the solution self-consistent.

\section{Hybridization functions: properties of the underlying two-impurity model}

To confirm the role of the VB-DMFT hybridization function in determining the transition we analyze its behavior at different dopings. In Figs. 18 and 19 we display $\Delta_{ \pm}(\omega)$ which appear in the self-consistent two-impurity Anderson model solved in VB-DMFT. The hybridization function for the even orbital $\Delta_{+}(\omega)$ shows a smooth structure with a broad peak about the chemical potential and little variation as a function of doping.

The hybridization of the odd-orbital $\Delta_{-}(\omega)$ (see Fig. (19) has almost exactly the same behavior as $A_{-}(\omega)$ up to a rescaling. At low doping, $\Delta_{-}(\omega)$ also displays a pseudogap. Therefore, the self-consistency of the VBDMFT equations leads to a very non-trivial two-impurity 


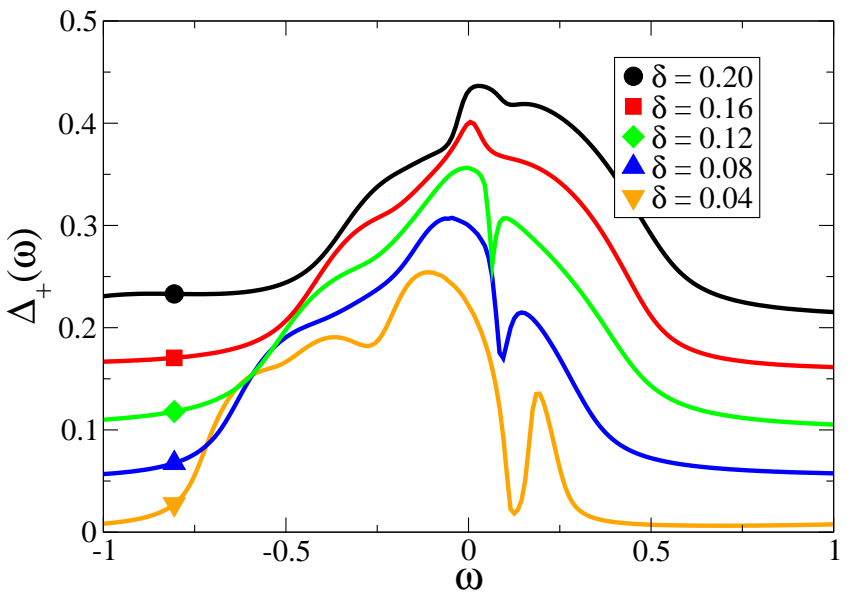

FIG. 18: (Color online) $\Delta_{+}(\omega)$ for the even orbital obtained with Padé approximants (see Appendix $\mathrm{B}$ ), for various dopings at $\beta=200$. A shift has been added between the curves for clarity.

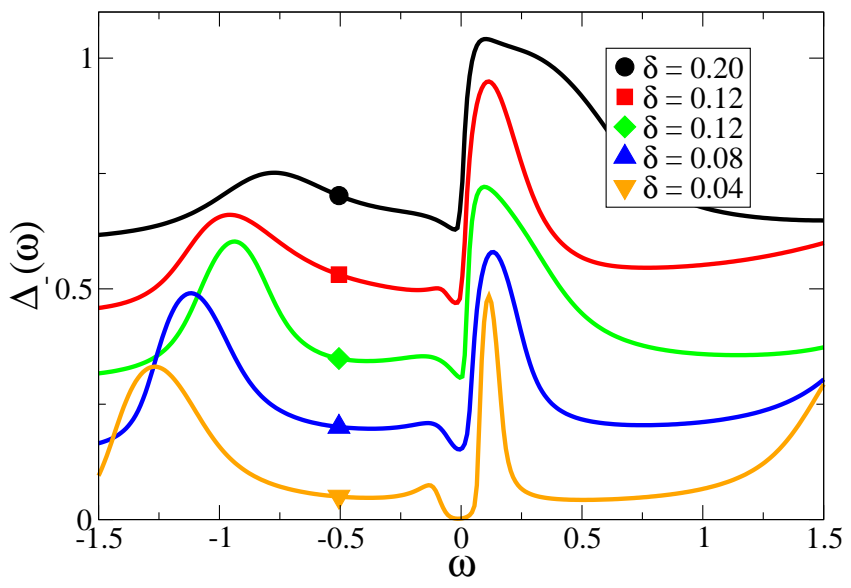

FIG. 19: (Color online) $\Delta_{-}(\omega)$ for the odd orbital obtained with Padé approximants (see Appendix B), for various dopings at $\beta=200$. A shift has been added between each curves for clarity

Anderson model in the low-doping regime: The evenorbital hybridization function is rather smooth but the odd orbital $\Delta_{-}(\omega)$ is pseudogapped.

It is interesting to note that around the critical doping, the low-energy part of $\Delta_{+}(\omega)$ becomes particle-hole symmetric, even though this is not the case for $A_{+}(\omega)$. This property is most clearly seen in the real part of $\Delta_{+}\left(i \omega_{n}\right)$ on the Matsubara axis (see Fig. 27) that nearly vanishes for $\delta$ between $12 \%$ and $16 \%$. This suggests a possible relation between the orbital-selective transition found in VB-DMFT and the critical point of the twoimpurity Kondo model $\stackrel{37,55}{5}$ The latter is known to exist only at particle-hole symmetry, but the self-consistency can in principle restore dynamically the symmetry and bring the system close to the critical point $\underline{38}$ These issues deserve further investigation and are left for future work.

\section{MOMENTUM-SPACE INTERPOLATION AND FERMI ARCS}

\section{A. Momentum-space reconstruction and comparison with larger clusters}

In this section, we address the problem of the reconstruction of momentum-space information starting from our valence-bond description. In doing so we will also address the reliability of our calculations by comparing our results with those obtained with larger clusters. This will provide a benchmark of our approach.

An important issue in theories that use clusters to describe lattice systems is how to infer quantities for the full lattice, starting from the information available from the finite-cluster calculation. In principle, this problem can be approached by a finite-size scaling study in order to extrapolate quantities in the thermodynamic limit. However, for cluster-DMFT theories this requires a huge computational effort, and the size of the clusters accessible to calculations is relatively small.

In order to obtain momentum-dependent quantities, it is necessary to employ some form of reconstruction based on the available cluster quantities. Indeed, DCA methods give direct access to lattice quantities (e.g. the selfenergy) only for a few special points in the Brillouin zone: the cluster momenta. In our simple description based on a single bond, they are $\mathbf{k}=(0,0)$ and $\mathbf{k}=(\pi, \pi)$. At these momenta, the lattice quantities can be unambiguously extracted from their cluster counterparts. For example, in our case: $\Sigma_{\text {latt }}(0,0)=\Sigma_{11}+\Sigma_{12}=\Sigma_{+}$and $\Sigma_{\text {latt }}(\pi, \pi)=\Sigma_{11}-\Sigma_{12}=\Sigma_{-}$. From the knowledge at these points, one would like to reconstruct any point in the Brillouin zone, using some interpolation procedure in order to avoid unphysical discontinuities of e.g. the self-energy in momentum space. Note that a similar procedure (reperiodization) must be used in the real-space cluster methods like CDMFT, $\stackrel{4}{\underline{4}}$ in order to restore the broken translation invariance. Clearly, there is some degree of arbitrariness associated with this procedure. The most important ingredient is the choice of the quantity to interpolate. Since cluster quantities describe accurately the short-range physics, it is expected that observables which are more local (short-range) in real-space (hence less $k$-dependent) are better suited for interpolation.

A standard method ( $\Sigma$-interpolation) in DCA calculation consists in interpolating the self-energy $\Sigma$ (see e.g. Ref. 58). In this paper, we choose a simple interpolation, in which the lattice self-energy is given by

$$
\Sigma_{\text {latt }}^{(\Sigma)}(\mathbf{k}, \omega)=\Sigma_{+}(\omega) \alpha_{+}(\mathbf{k})+\Sigma_{-}(\omega) \alpha_{-}(\mathbf{k}),
$$

with $\alpha_{ \pm}(\mathbf{k})=\frac{1}{2}\left\{1 \pm \frac{1}{2}\left[\cos \left(k_{x}\right)+\cos \left(k_{y}\right)\right]\right\}$. By rewriting this in terms of the on-site $\left(\Sigma_{11}(\omega)\right)$ and inter-site $\left(\Sigma_{12}(\omega)\right)$ components of the cluster self-energy

$$
\Sigma_{\text {latt }}^{(\Sigma)}(\mathbf{k}, \omega)=\Sigma_{11}(\omega)+\frac{1}{2} \Sigma_{12}(\omega)\left[\cos \left(k_{x}\right)+\cos \left(k_{y}\right)\right] .
$$


This can be viewed as a truncation of the Fourier expansion of the lattice self-energy to the first two Fourier components. Note that, the nearest-neighbor component of the lattice self-energy is obtained, according to this formula, as $\Sigma_{n n}=\Sigma_{12} / 4$, which is analogous to the reperiodization procedure of CDMFT (see e.g. Ref. 4).

Another method ( $M$-interpolation) has been recently introduced in Ref. 59,60 in the CDMFT method. It consists in interpolating the cumulant, defined as $M \equiv$ $(\omega+\mu-\Sigma)^{-1}$. The lattice cumulant is obtained as

$M_{\text {latt }}(\mathbf{k}, \omega)=\alpha_{+}(\mathbf{k}) \frac{1}{\omega+\mu-\Sigma_{+}(\omega)}+\alpha_{-}(\mathbf{k}) \frac{1}{\omega+\mu-\Sigma_{-}(\omega)}$.

From $M_{\text {latt }}(\mathbf{k}, \omega)$ it is then possible to extract a lattice self-energy by

$$
\Sigma_{\text {latt }}^{(M)}(\mathbf{k}, \omega)=\omega+\mu-M_{\text {latt }}(\mathbf{k}, \omega)^{-1} .
$$

The cumulant is the dual quantity of the self-energy in an expansion around the atomic limit. It is a natural measure of how much the hybridization to the self-consistent environment changes the impurity Green's function as compared to an isolated dimer.

The $\Sigma$-interpolation is based on the assumption that the self-energy is sufficiently short-range or small enough for all frequencies. It corresponds to an expansion around the free-electron limit, hence it is expected to work better at weak coupling. On the other hand, the $M$ interpolation is expected to be better close to the atomic limit, and more generally at strong coupling, for example close to a Mott insulating state where the cumulant is more local than the self-energy ${ }^{59}$ Other methods, like the periodization of the Green's function ${ }^{25}$ have also been discussed, in the CDMFT context. In this section, we focus on a quantitative comparison of the $\Sigma$-interpolation and the $M$-interpolation, using a plaquette (4 sites) calculation as a benchmark.

Let us emphasize again that for our two-site cluster the momenta $\mathbf{k}=(0,0)$ and $\mathbf{k}=(\pi, \pi)$ are special. At these two points lattice quantities are independent of the interpolation method used (since at those momenta, one of the $\alpha$ 's vanishes), while at all other momenta the quantities reconstructed with the two methods differ. On the other hand, in a four-site cluster (plaquette) approach, there are two additional momenta where the description is unbiased by the interpolation procedure, namely $\mathbf{k}=(0, \pi)$ and $\mathbf{k}=(\pi, 0)$, which are equivalent if rotational symmetry is not broken. Hence performing a plaquette calculation gives us the opportunity to compare directly cluster self-energies obtained with the dimer and the plaquette at momenta $\mathbf{k}=(0,0)$ and $\mathbf{k}=(\pi, \pi)$, and furthermore provides a test for the interpolation method by comparing self-energies at $\mathbf{k}=(0, \pi)$.

We compare in Fig. 20 the results of VB-DMFT and plaquette calculations for momenta $\mathbf{k}=(0,0)$ and $\mathbf{k}=$ $(\pi, \pi)$ for $\delta=8 \%$ (upper panel) and $\delta=16 \%$ (lower panel), in Matsubara frequencies. The agreement between the two cluster calculations is good. The descriptions of the Hubbard model given by VB-DMFT and plaquette cluster calculations are consistent with one another for these momenta. In order to decide which momentum-interpolation procedure is better within VBDMFT, we also compare in Fig. 21 (at $\delta=8 \%$ in the upper panel and $\delta=16 \%$ in the lower panel) the selfenergy obtained from the $\Sigma$ - and $M$ - interpolations, at momentum $\mathbf{k}=(0, \pi)$, to the self-energy obtained from a direct plaquette calculation (for which $(0, \pi)$ is a cluster momentum). Comparing the two data sets, we see that the $M$-interpolation is clearly superior to the $\Sigma$ interpolation in reconstructing the self-energy at $(0, \pi)$.
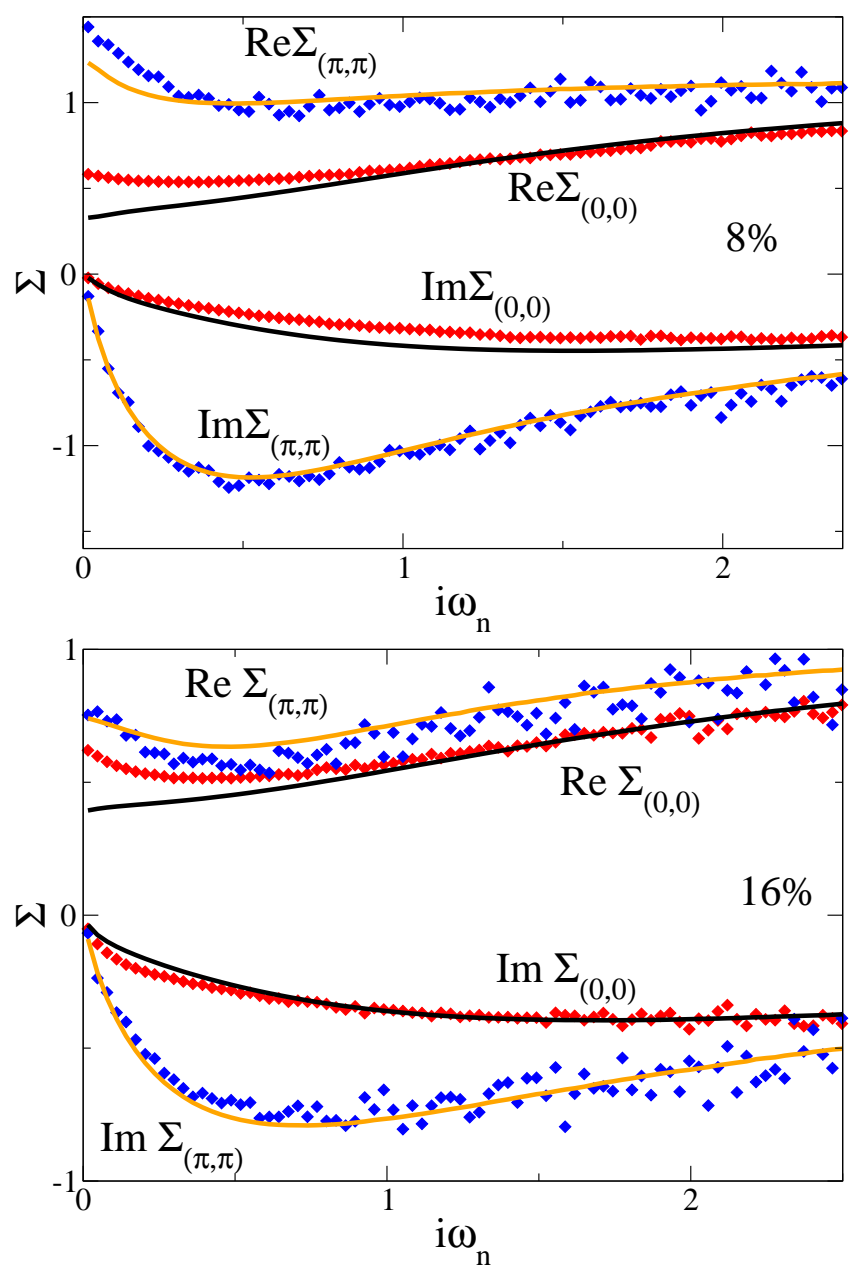

FIG. 20: (Color online) Real and imaginary part of dimer (solid lines) and plaquette (symbols) self-energies at $k=(0,0)$ (black solid line and red diamond) and $k=(\pi, \pi)$ (orange solid line and blue diamond) for $\beta=200$ and $\delta=0.08$ (upper panel) and $\delta=0.16$ (lower panel).

Applying the $M$-interpolation to the VB-DMFT results we can qualitatively, and to a large extent quantitatively, reproduce the larger cluster (plaquette) results, hence providing a justification to the use of the $M$-interpolation. It is important to stress that the plaquette cluster-momentum $\mathbf{k}=(0, \pi)$ is not present as an individual orbital in the two-site description: It is entirely 

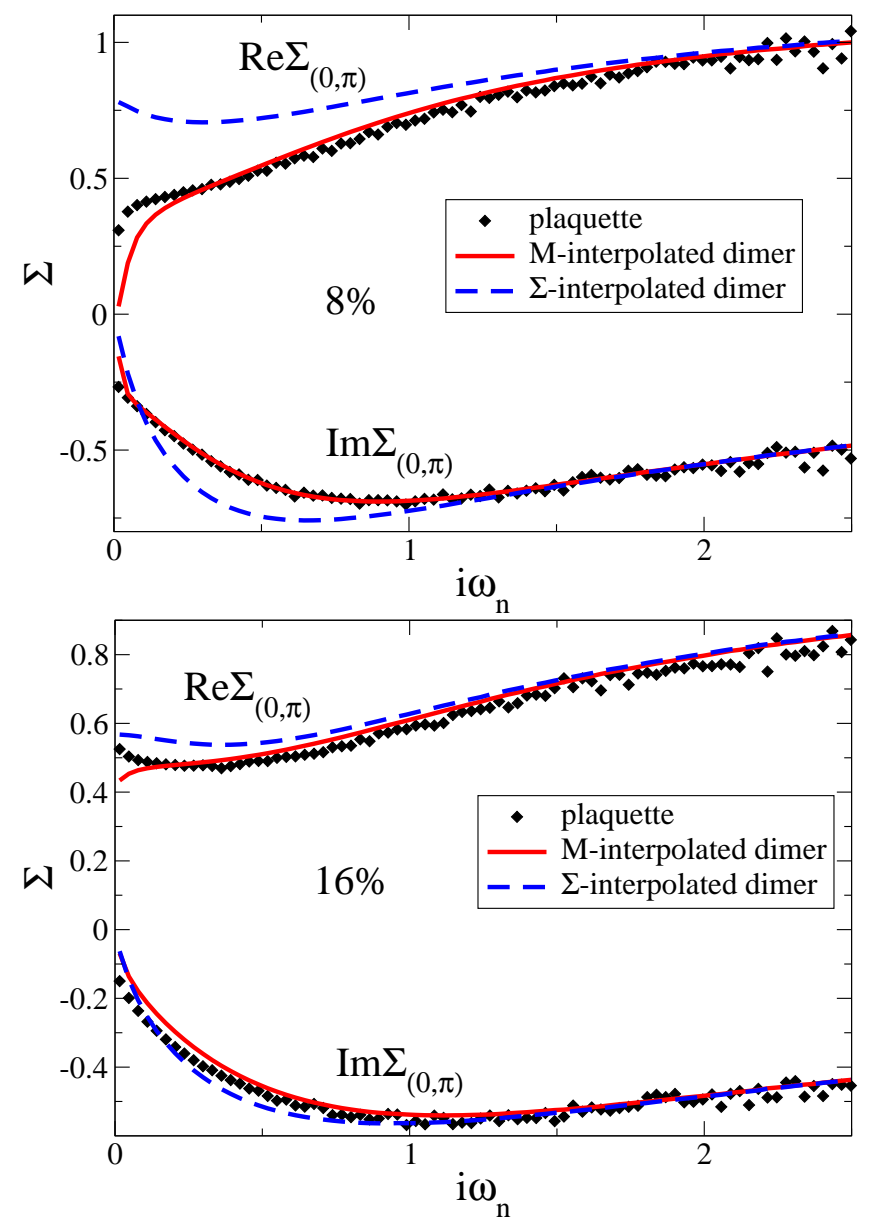

FIG. 21: (Color online) Comparison between the reconstructed $\Sigma_{(0, \pi)}$ using $M$-interpolation (red solid line) and $\Sigma$ interpolation (blue dashed line) in the dimer and the cluster self-energy of the plaquette calculation at $k=(0, \pi)$ (black diamonds), for $\delta=0.08$ (upper panel) and $\delta=0.16$ (lower panel) . $\beta=200$.

reconstructed by interpolation, and as such is the most direct test of the reconstructed momentum dependence.

\section{B. Fermi arcs and momentum differentiation}

We can now study momentum differentiation using the $M$-interpolation. As we shall see, VB-DMFT indeed provides a simple description of momentum differentiation as observed in ARPES experiments. This is illustrated by the intensity maps of the spectral function $A(\mathbf{k}, 0)$ displayed in Fig. 22. At very high doping $\delta \gtrsim 25 \%$ (not shown), cluster corrections to DMFT are negligible and the spectral intensity is uniform along the Fermi surface. In contrast, as the doping level is reduced, momentum differentiation sets in around the characteristic doping at which the localization of the outer orbital takes place. The intensity maps then display apparent "Fermi arcs" at finite temperature with higher spectral intensity in the
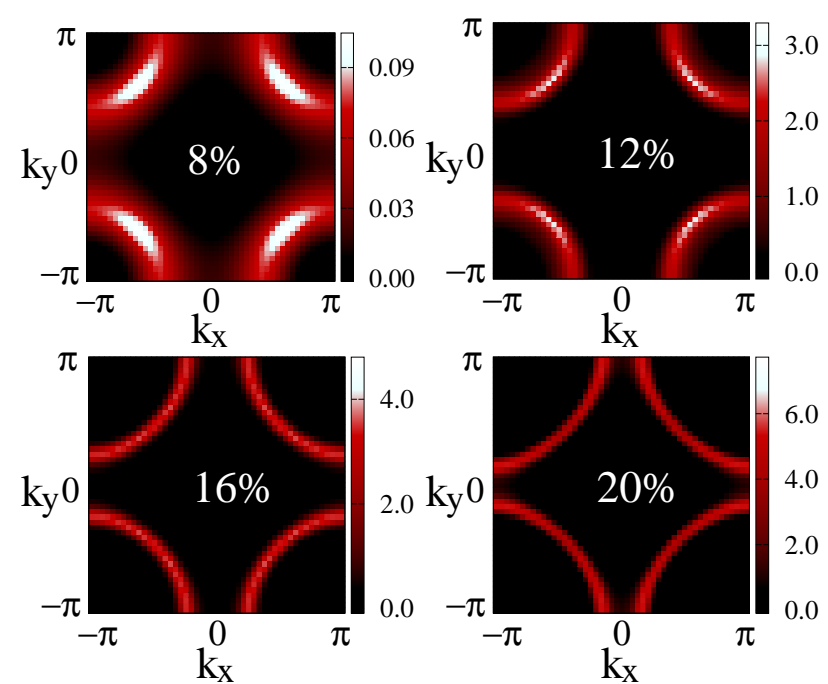
.

FIG. 22: (Color online) Intensity maps of the spectral function $A(\mathbf{k}, 0)$ for different doping levels obtained with $M$ interpolation.

nodal direction in comparison to antinodes, in qualitative agreement with experiments (see e.g. Refs. 13,61) and earlier CDMFT calculations with larger clusters $\underline{23,24,29}$ The mechanism behind the suppression of spectral weight at the antinodes at low doping is clearly associated, in our results, to Mott localization and the importance of singlet correlations. In technical terms, this is associated with the large real part in $\Sigma_{-}=\Sigma(\pi, \pi)$ (cf. Fig. 3), which induces a pseudogap in the antinodal orbital, and with the large imaginary part of the self-energy in the $(\pi, 0)$ and $(\pi, \pi)$ regions, which also contribute to the suppression of spectral weight in the antinodal region.
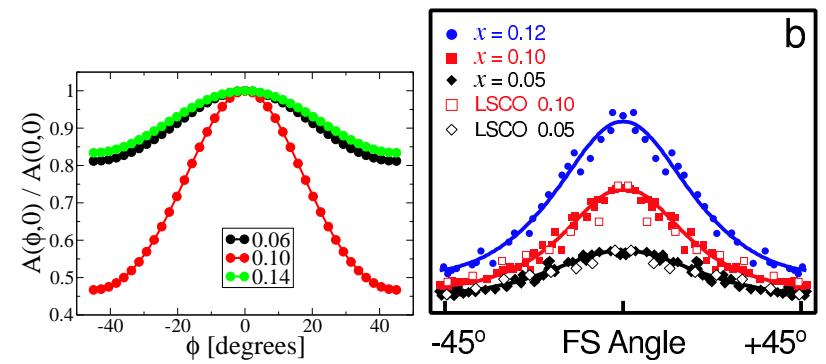

FIG. 23: (Color online) Left panel: Normalized intensity $A(\phi, 0) / A(0,0)$ along the Fermi surface vs the angle to the diagonal of the Brillouin zone in degrees $(\phi=0$ is the node, $\phi= \pm 45$ the antinode). The nodal intensity $A(0,0)$ is 0.045 for $\delta=6 \%, 1.66$ for $\delta=10 \%$ and 4.61 for $\delta=14 \%$. $\quad \beta=200$. Right panel: Angular dependence of the spectral weight along the Fermi Surface in $\mathrm{Ca}_{2-x} \mathrm{Na}_{x} \mathrm{CuO}_{2} \mathrm{Cl}_{2}$ at $x=0.05$ (black diamonds), $x=0.10$ (red squares), and $x=0.12$ (blue circles) along with data from $\mathrm{La}_{2-x} \mathrm{Sr}_{x} \mathrm{CuO}_{4}$ for $x=0.05$ and $x=0.10$ (open symbols). Figure reprinted from Ref. 61 (Fig. 3b). Copyright 2005 by Science.

In order to compare this momentum-space differentiation to experiments in a more quantitative manner, 
we plot in Fig. 23 the contrast of the spectral intensity along the Fermi surface for different doping levels. This plot compares very favorably to the experimental data of Ref. 61, which are also reproduced for convenience. In particular, we observe that the differentiation has a non-monotonous behavior, reaching a maximum around $\delta \approx 10 \%$, and then decreasing for lower dopings.

VB-DMFT is able to capture momentum differentiation reliably for doping levels between $10 \%$ and $20 \%$. For very low doping $(\delta \lesssim 8 \%)$ the $M$-interpolated self-energy develops singularities on lines in momentum space, leading to lines of zeroes of the Green's function and to the breakup of the Fermi surface $\underline{59,62,63,64,65}$ It would be very instructive to relate this with recent quantum oscillation experiments ${ }^{66}$ However, in this regime, a better momentum resolution (larger clusters) is necessary to obtain reliable results.

\section{CONCLUSION}

In this paper, we presented in detail the valence-bond DMFT approach to correlated electrons ${ }^{34}$ and used it to treat the two-dimensional Hubbard model with nearestneighbor and next-nearest neighbor hopping. The approach reduces to single-site DMFT when intersite correlations are unimportant. This is the case at large doping levels. Near the Mott transition, at lower doping levels, these correlations dominate the physics and lead to the phenomenon of momentum-space differentiation.

This phenomenon corresponds to the destruction of coherent quasiparticle excitations in the antinodal regions of the Brillouin zone. In those regions, a pseudogap opens and quasiparticles become increasingly incoherent as the doping level is reduced. In contrast, in nodal regions, quasiparticles are protected and their lifetime actually increases as the pseudogap opens. The physics of the low-doping regime is dominated by strong singlet correlations between nearest-neighbor sites.

VB-DMFT is a minimal cluster description of a lowdimensional strongly-correlated system in terms of two effective degrees of freedom, associated to each of the important regions in momentum space (nodal and antinodal). These two degrees of freedom are treated as the two orbitals of an effective dimer impurity model. Momentum-space differentiation emerges as an orbitalselective Mott transition in which a pseudogap opens in the spectrum of the antinodal degree of freedom, while the nodal one remains a coherent Fermi liquid. The simplicity of the approach allows for a highly accurate numerical solution of the VB-DMFT equations. It also allows for the use of a semi-analytical technique, the rotationally-invariant slave-bosons method, as an approximate impurity solver.

Comparisons of the results of VB-DMFT and plaquette calculations put the cluster extensions of DMFT on much firmer footing. It has been known for a while that in the high-temperature and high-enough doping regime, single- site DMFT is very accurate and cluster corrections are quantitatively small. On the other hand, the validity of cluster extensions of DMFT in the underdoped regime, where momentum-space differentiation is strong, is not as universally accepted. Consistency of the results between 2 -site calculations and calculations with larger clusters provides support to the validity of cluster approaches.

The qualitative picture that emerges from VB-DMFT is in excellent qualitative agreement with photoemission results in the normal state of the copper-oxide based high-temperature superconductors. The selective destruction of quasiparticles at the antinodes is associated with the 'Fermi arcs' observed in ARPES. Comparison of the evolution of the tunneling density of states with temperature against experimental data is also encouraging. Many more detailed comparisons of VB-DMFT against various other spectroscopies should be carried out in future work in order to determine the strengths and the limitations of the method and in order to further advance our understanding and our ability to capture with simple models some of the physical properties of cuprates.

\section{Acknowledgments}

We thank C. Berthod, J. C. Campuzano, L. de' Medici, J. C. Seamus Davis, M. Fabrizio, K. Haule, F. Lechermann, A. J. Millis, T. M. Rice, A. Sacuto and P. Werner for useful discussions. We also thank F. Assaad and M. Aichhorn for providing results using the stochastic maximum entropy method. We acknowledge support from The Partner University Fund (PUF-FACE), ICAM, the ANR (under grants ETSF, GASCOR and ECCE) and the NSF-Materials World Network. One of us (PSC) thanks CPHT and IPhT-Saclay for hospitality.

\section{APPENDIX A: DCA WITH BRILLOUIN-ZONE PATCHES OF ARBITRARY SHAPES}

In this appendix, we review for completeness the basic formalism of DCA, 18,67,68 and discuss in particular its extension to Brillouin-zone patches with equal volume and arbitrary shape.

DCA can be seen as an approximation to the Luttinger-Ward (LW) functional of a lattice theory. In the LW functional the conservation of momentum at the vertex of diagrams is accounted for by the function

$$
\Delta\left(\mathbf{k}_{1}, \mathbf{k}_{2}, \mathbf{k}_{3}, \mathbf{k}_{4}\right)=N \delta_{\mathbf{k}_{1}+\mathbf{k}_{2}, \mathbf{k}_{3}+\mathbf{k}_{4}} .
$$

In single-site DMFT momentum conservation at the internal vertices of the diagrams is ignored and $\Delta \equiv 1$. DCA attempts at partially restoring momentum conservation by partitioning the Brillouin zone into $N_{p}$ patches $\mathcal{P}_{\mathbf{K}}$ centered around a subgroup of $N_{p}$ momenta $\mathbf{K}$ and approximating the momentum conservation with

$$
\Delta\left(\mathbf{k}_{1}, \mathbf{k}_{2}, \mathbf{k}_{3}, \mathbf{k}_{4}\right) \sim N_{p} \delta_{\mathbf{K}_{1}+\mathbf{K}_{2}, \mathbf{K}_{3}+\mathbf{K}_{4}},
$$


where $\mathbf{K}_{i}$ is the representative vector of the patch containing $\mathbf{k}_{i}$. This corresponds to taking into account momentum conservation among the patches and discarding momentum conservation inside the single patch.

The DCA LW functional contains the same diagrams as the original lattice functional with all the internal Green's functions replaced by the coarse-grained Green's functions

$$
G(\mathbf{K})=\frac{1}{N_{K}} \sum_{\mathbf{k} \in \mathcal{P}_{\mathbf{K}}} G_{\text {latt }}(\mathbf{k}),
$$

where $N_{K}$ is the number of momenta contained in the patch $\mathcal{P}_{\mathbf{K}}$ (the volume of the patch). To see this we can consider the simplest graph contributing to the LW functional. The contribution of this graph to the lattice functional is given by

$$
\begin{aligned}
\Phi_{\text {latt }}= & \frac{1}{N^{4}} \sum_{\mathbf{k}_{1} \mathbf{k}_{2} \mathbf{k}_{3} \mathbf{k}_{4}} G_{\text {latt }}\left(\mathbf{k}_{1}\right) G_{\text {latt }}\left(\mathbf{k}_{2}\right) G_{\text {latt }}\left(\mathbf{k}_{3}\right) G_{\text {latt }}\left(\mathbf{k}_{4}\right) \\
U^{2} N^{2} \delta_{\mathbf{k}_{1}+\mathbf{k}_{2}, \mathbf{k}_{3}+\mathbf{k}_{4} .} . & \text { (A4) }
\end{aligned}
$$

Replacing the original momentum conservation with the DCA approximation we obtain

$$
\begin{aligned}
\Phi_{\mathrm{DCA}}= & \frac{1}{N^{4}} \sum_{\mathbf{K}_{i}} \sum_{\tilde{\mathbf{k}}_{i}} G_{\text {latt }}\left(\mathbf{K}_{1}+\tilde{\mathbf{k}}_{1}\right) G_{\text {latt }}\left(\mathbf{K}_{2}+\tilde{\mathbf{k}}_{2}\right) \\
& \times G_{\text {latt }}\left(\mathbf{K}_{3}+\tilde{\mathbf{k}}_{3}\right) G_{\text {latt }}\left(\mathbf{K}_{4}+\tilde{\mathbf{k}}_{4}\right) \\
& U^{2} N_{p}^{2} \delta_{\mathbf{K}_{1}+\mathbf{K}_{2}, \mathbf{K}_{3}+\mathbf{K}_{4}} \\
= & \sum_{\mathbf{K}_{i}} \frac{N_{K_{1}} N_{K_{2}} N_{K_{3}} N_{K_{4}}}{N^{4}} \\
& \left(\frac{1}{N_{K_{1}}} \sum_{\tilde{\mathbf{k}}_{1}} G_{\text {latt }}\left(\mathbf{K}_{1}+\tilde{\mathbf{k}}_{1}\right)\right) \times \\
& \times \ldots \times\left(\frac{1}{N_{K_{4}}} \sum_{\tilde{\mathbf{k}}_{4}} G_{\text {latt }}\left(\mathbf{K}_{4}+\tilde{\mathbf{k}}_{4}\right)\right) \\
& U^{2} N_{p}^{2} \delta_{\mathbf{K}_{1}+\mathbf{K}_{2}, \mathbf{K}_{3}+\mathbf{K}_{4}} \\
= & \sum_{\mathbf{K}_{i}} \frac{N_{K_{1}} N_{K_{2}} N_{K_{3}} N_{K_{4}}}{N^{4}} G\left(\mathbf{K}_{1}\right) \ldots G\left(\mathbf{K}_{4}\right) \\
& N_{p}^{2} U^{2} \delta_{\mathbf{K}_{1}+\mathbf{K}_{2}, \mathbf{K}_{3}+\mathbf{K}_{4}} .
\end{aligned}
$$

It is then clear that if the number of $\mathbf{k}$ - points (the BZ volume) is the same for every patch, the prefactor can be simplified, yielding

$$
\begin{aligned}
\Phi_{\mathrm{DCA}}= & \frac{1}{N_{p}^{4}} \sum_{\mathbf{K}_{i}} G\left(\mathbf{K}_{1}\right) \ldots G\left(\mathbf{K}_{4}\right) \\
& N_{p}^{2} U^{2} \delta_{\mathbf{K}_{1}+\mathbf{K}_{2}, \mathbf{K}_{3}+\mathbf{K}_{4} .}
\end{aligned}
$$

This functional corresponds to the functional of a problem with $N_{p}$ momenta and hence can be obtained by the solution of a cluster impurity problem. The crucial observation is that, as long as the volume is the same for all the patches, the DCA functional is the functional of a cluster problem which, once expressed in real space coordinates, retains purely local interactions, precisely identical to those of the original Hubbard model. This ensures that this procedure does not generate additional interactions in the cluster.

However, the shape of the patches is not constrained in this procedure. A possible route to exploit this freedom is to notice that DCA can also be interpreted as an approximation to the lattice self-energy (see Sec. IIA). Indeed DCA corresponds to approximating the self-energy by a constant value in each patch

$$
\Sigma\left(\mathbf{K}_{i}+\tilde{\mathbf{k}}, \omega\right) \sim \Sigma\left(\mathbf{K}_{i}, \omega\right) \quad \forall \mathbf{K}_{i}+\tilde{\mathbf{k}} \in \mathcal{P}_{\mathbf{K}_{i}} .
$$

Hence using physical intuition patches can be shaped in such a way to enclose regions with definite properties, such as nodal and antinodal regions for example.

\section{APPENDIX B: ANALYTICAL CONTINUATIONS USING PADÉ APPROXIMANTS}

In order to perform the analytical continuations shown earlier in this paper, we have used $N$-point Padé approximants $\frac{42}{4}$

$$
C_{N}(z)=A_{N}(z) / B_{N}(z),
$$

where $A_{N}$ and $B_{N}$ are polynomials of order $(N-1) / 2$ and $(N-1) / 2$ if $N$ is odd and $(N-2) / 2$ and $N / 2$ if $N$ is even. The Padé approximant $C_{N}$ can alternatively be written as a continued fraction

$$
C_{N}(z)=\frac{a_{1}}{1+} \frac{a_{2}\left(z-z_{1}\right)}{1+} \ldots \frac{a_{N}\left(z-z_{N-1}\right)}{1} .
$$

The polynomials $A_{N}$ and $B_{N}$ are then given by a recursion formula

$$
\begin{gathered}
A_{n+1}(z)=A_{n}(z)+\left(z-z_{n}\right) a_{n+1} A_{n-1}(z) \\
B_{n+1}(z)=B_{n}(z)+\left(z-z_{n}\right) a_{n+1} B_{n-1}(z),
\end{gathered}
$$

with

$$
A_{0}=0, \quad A_{1}=a_{1}, \quad B_{0}=B_{1}=1 .
$$

In order to construct the complex function $C_{N}(z)$, we impose that it is equal to the function $f$ to be continued on the real axis at the first $N$ Matsubara frequencies

$$
C_{N}\left(i \omega_{n}\right)=f\left(i \omega_{n}\right) \quad \forall n=1, \ldots, N .
$$

This constraint can be achieved by determining the coefficients $a_{n}$ with the recursion

$$
\begin{array}{r}
a_{n}=g_{n, n}, \quad g_{1, n}=f\left(i \omega_{n}\right), \quad n=1, \ldots, N \\
g_{p, q}=\frac{g_{p-1, p-1}-g_{p-1, q}}{\left(i \omega_{q}-i \omega_{p-1}\right) g_{p-1, q}} .
\end{array}
$$

In this paper, the Monte Carlo data on the Matsubara axis has been averaged over $5 \times 10^{8}$ measures. A Padé 
approximant was computed for $\Sigma_{ \pm}$imposing that they match over the first 200 Matsubara frequencies for a temperature $1 / \beta=1 / 200$. The self-energies $\Sigma_{ \pm}(\omega)$ obtained on the real-frequency axis using this procedure were then used to compute the spectral functions through

$$
A_{ \pm}(\omega)=-\frac{1}{\pi} \operatorname{Im} \sum_{\mathbf{k} \in \mathcal{P}_{ \pm}} \frac{1}{\omega+\mu-\varepsilon_{\mathbf{k}}-\Sigma_{ \pm}(\omega)} .
$$

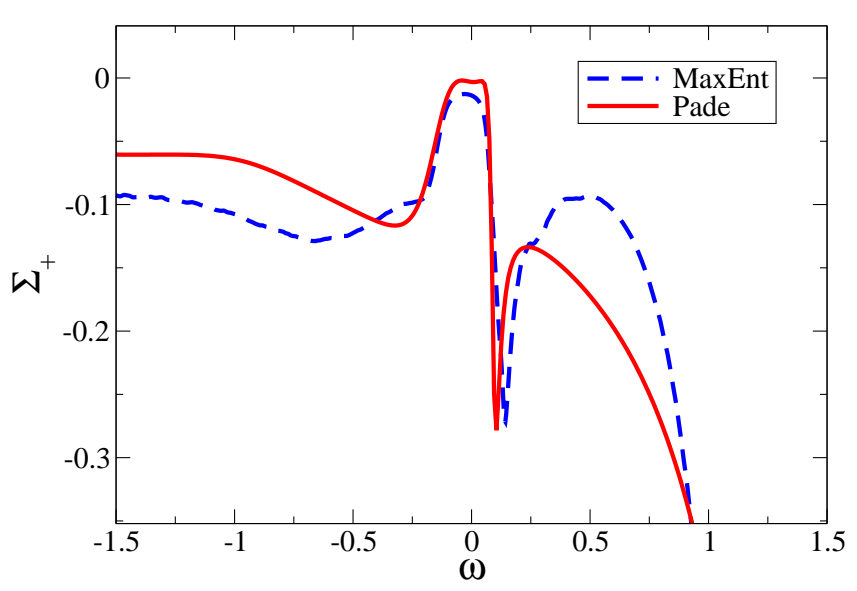

FIG. 24: (Color online) Even self-energy on the real frequency axis as obtained using Padé approximants (solid line) and by maximum entropy (dashed line). $\delta=8 \%$ and $\beta=200$.
In order to test the quality of the analytical continuations, we constructed a Padé approximant for several independent runs checking that they lead to qualitatively similar results. Indeed, spurious poles sometimes appear on the real-frequency axis producing unphysical results. We also compared the Padé approximant with the outcome of a stochastic maximum entropy method ${ }^{69.70} \mathrm{~A}$ typical outcome is shown in Fig. 24. Even if both method lead to results that are slightly different at a quantitative level, they display the same main qualitative features. Therefore all our physical conclusions stated earlier do not depend on the analytical continuation method.

\section{APPENDIX C: RAW CTQMC DATA}

For completeness, we display the raw CTQMC data on the Matsubara axis for the Green's functions $G_{ \pm}$, the selfenergies $\Sigma_{ \pm}$and the hybridization functions $\Delta_{ \pm}$. The analytical continuations using Padé approximants (see Appendix (B) have been performed on this data.
1 P. W. Anderson, Science 235, 1196 (1987).

${ }^{2}$ W. F. Brinkman and T. M. Rice, Phys. Rev. B 2, 4302 (1970).

3 A. Georges, G. Kotliar, W. Krauth, and M. J. Rozenberg, Rev. Mod. Phys. 68, 13 (1996).

${ }^{4}$ G. Kotliar, S. Y. Savrasov, K. Haule, V. S. Oudovenko, O. Parcollet, and C. A. Marianetti, Rev. Mod. Phys. 78, 865 (2006).

${ }^{5}$ G. Kotliar and D. Vollhardt, Physics Today March 2004, 53 (2004).

${ }^{6}$ G. Baskaran, Z. Zou, and P. W. Anderson, Sol. State Comm. 63, 973 (1987).

7 G. Kotliar and J. Liu, Phys. Rev. B 38, 5142 (1988).

8 M. Grilli and G. Kotliar, Phys. Rev. Lett. 64, 1170 (1990).

9 Y. Suzumura, Y. Hasegawa, and H. Fukuyama, J. Phys. Soc. Jap. 57, 2768 (1988).

10 P. A. Lee, N. Nagaosa, and X.-G. Wen, Rev. Mod. Phys. 78, 17 (2006).

11 C. Gros, Phys. Rev. B 38, 931 (1988).

12 A. Paramekanti, M. Randeria, and N. Trivedi, Phys. Rev. Lett. 87, 217002 (2001).

13 A. Damascelli, Z. Hussain, and Z.-X. Shen, Rev. Mod. Phys. 75, 473 (2003).

14 M. Le Tacon, A. Sacuto, A. Georges, G. Kotliar, Y. Gallais, D. Colson, and A. Forget, Nat. Phys. 2, 537 (2006).

15 F. Venturini, M. Opel, T. P. Devereaux, J. K. Freericks, I. Tütt, B. Revaz, E. Walker, H. Berger, L. Forró, and R. Hackl, Phys. Rev. Lett. 89, 107003 (2002).

16 W. Guyard, M. L. Tacon, M. Cazayous, A. Sacuto,
A. Georges, D. Colson, and A. Forget, Phys. Rev. B 77, 024524 (2008).

17 Y. Kohsaka, C. Taylor, P. Wahl, A. Schmidt, J. Lee, K. Fujita, J. W. Alldredge, K. McElroy, J. Lee, H. Eisaki, et al., Nature 454, 1072 (2008).

18 T. Maier, M. Jarrell, T. Pruschke, and M. H. Hettler, Rev. Mod. Phys. 77, 1027 (2005).

19 A.-M. S. Tremblay, B. Kyung, and D. Sénéchal, Low Temp. Phys. 32, 424 (2006).

20 A. I. Lichtenstein and M. I. Katsnelson, Phys. Rev. B 62, R9283 (2000).

21 T. Maier, M. Jarrell, T. Pruschke, and J. Keller, Phys. Rev. Lett. 85, 1524 (2000).

22 M. Jarrell, T. Maier, M. H. Hettler, and A. N. Tahvildarzadeh, Europhys. Lett. 56, 563 (2001).

23 O. Parcollet, G. Biroli, and G. Kotliar, Phys. Rev. Lett. 92, 226402 (2004).

24 M. Civelli, M. Capone, S. S. Kancharla, O. Parcollet, and G. Kotliar, Phys. Rev. Lett. 95, 106402 (2005).

25 B. Kyung, S. S. Kancharla, D. Sénéchal, A.-M. S. Tremblay, M. Civelli, and G. Kotliar, Phys. Rev. B 73, 165114 (2006).

26 G. Biroli, O. Parcollet, and G. Kotliar, Phys. Rev. B 69, 205108 (2004).

27 K. Haule and G. Kotliar, Phys. Rev. B 76, 092503 (2007).

28 M. Capone and G. Kotliar, Phys. Rev. B 74, 054513 (2006).

29 K. Haule and G. Kotliar, Phys. Rev. B 76, 104509 (2007).

30 K. Haule and G. Kotliar, Europhys. Lett. 77, 27007 (2007). 

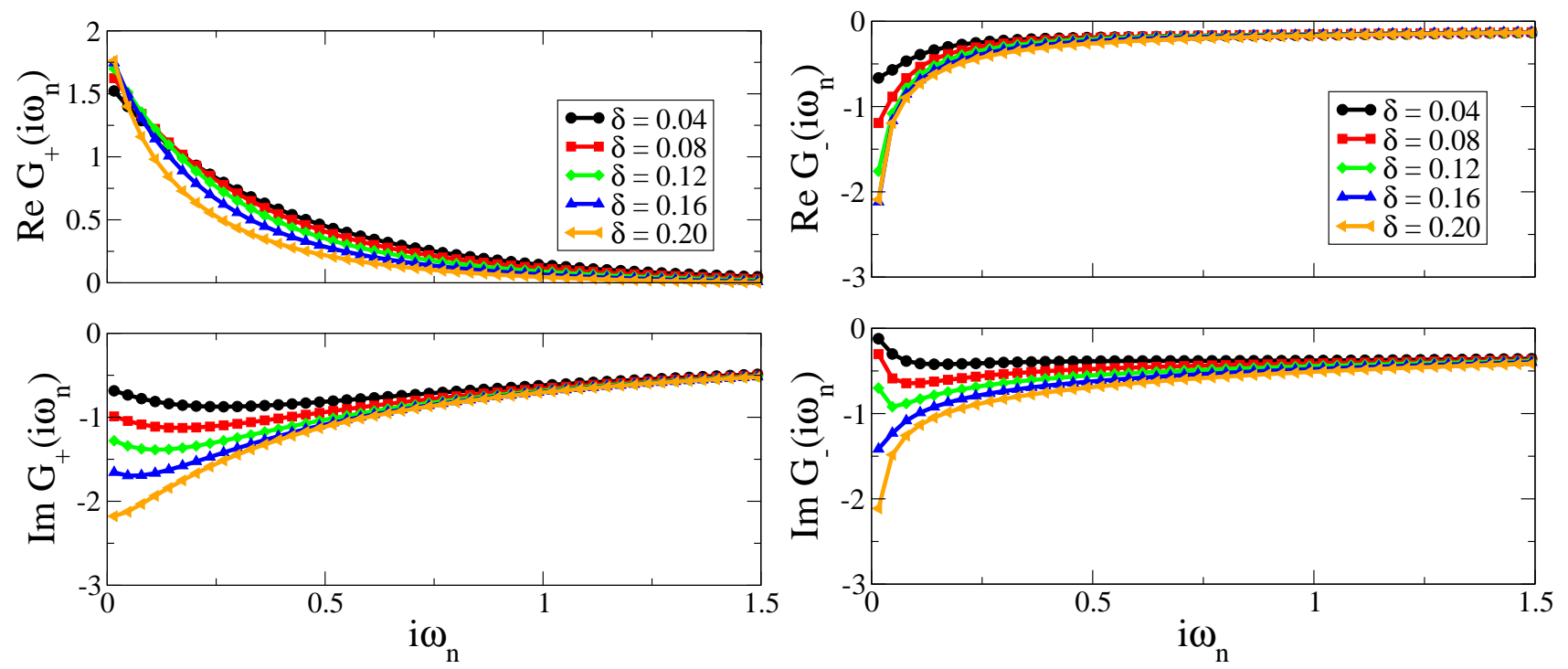

FIG. 25: (Color online) Even and odd Green's functions $G_{ \pm}\left(i \omega_{n}\right)$ on the Matsubara axis for various dopings at $\beta=200$.
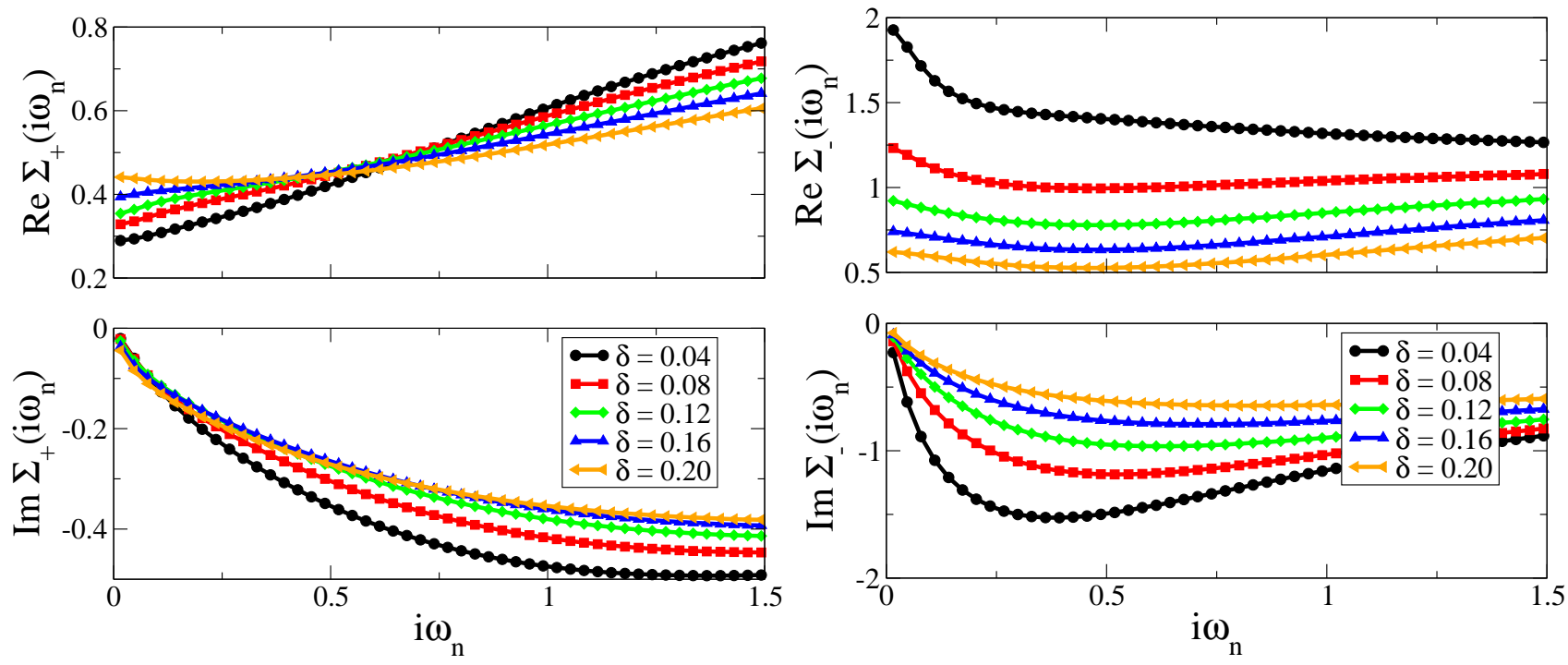

FIG. 26: (Color online) Even and odd self-energies $\Sigma_{ \pm}\left(i \omega_{n}\right)$ on the Matsubara axis for various dopings at $\beta=200$.

31 M. Civelli, M. Capone, A. Georges, K. Haule, O. Parcollet, T. D. Stanescu, and G. Kotliar, Phys. Rev. Lett. 100, 046402 (2008).

32 E. Gull, P. Werner, X. Wang, M. Troyer, and A. J. Millis, Europhys. Lett. 84, 37009 (2008).

33 T. A. Maier, M. Jarrell, T. C. Schulthess, P. R. C. Kent, and J. B. White, Phys. Rev. Lett. 95, 237001 (2005).

${ }^{34}$ M. Ferrero, P. S. Cornaglia, L. De Leo, O. Parcollet, G. Kotliar, and A. Georges, Europhys. Lett. (2009-to appear), arXiv:0806.4383.

35 S. Biermann, L. de' Medici, and A. Georges, Phys. Rev. Lett. 95, 206401 (2005).

${ }^{36}$ V. I. Anisimov, I. A. Nekrasov, D. E. Kondakov, T. M. Rice, and M. Sigrist, Eur. Phys. J. B 25, 191 (2002).

37 B. A. Jones, C. M. Varma, and J. W. Wilkins, Phys. Rev. Lett. 61, 125 (1988).

${ }^{38}$ M. Ferrero, L. De Leo, P. Lecheminant, and M. Fabrizio,
J. Phys.: Condens. Matter 19, 433201 (2007).

39 P. Werner, A. Comanac, L. de' Medici, M. Troyer, and A. J. Millis, Phys. Rev. Lett. 97, 076405 (2006).

${ }^{40}$ K. Haule, Phys. Rev. B 75, 155113 (2007).

41 F. Lechermann, A. Georges, G. Kotliar, and O. Parcollet, Phys. Rev. B 76, 155102 (2007).

${ }^{42}$ H. J. Vidberg and J. W. Serene, J. Low Temp. Phys. 29, 179 (1977).

43 S. E. Barnes, J. Phys. F: Metal Physics 6, 1375 (1976).

44 P. Coleman, Phys. Rev. B 29, 3035 (1984).

45 G. Kotliar and A. E. Ruckenstein, Phys. Rev. Lett. 57, 1362 (1986).

46 T. Li, P. Wölfle, and P. J. Hirschfeld, Phys. Rev. B 40, 6817 (1989).

47 M. Fabrizio, Phys. Rev. B 76, 165110 (2007).

$48 \varnothing$. Fischer, M. Kugler, I. Maggio-Aprile, C. Berthod, and C. Renner, Rev. Mod. Phys. 79, 353 (2007). 

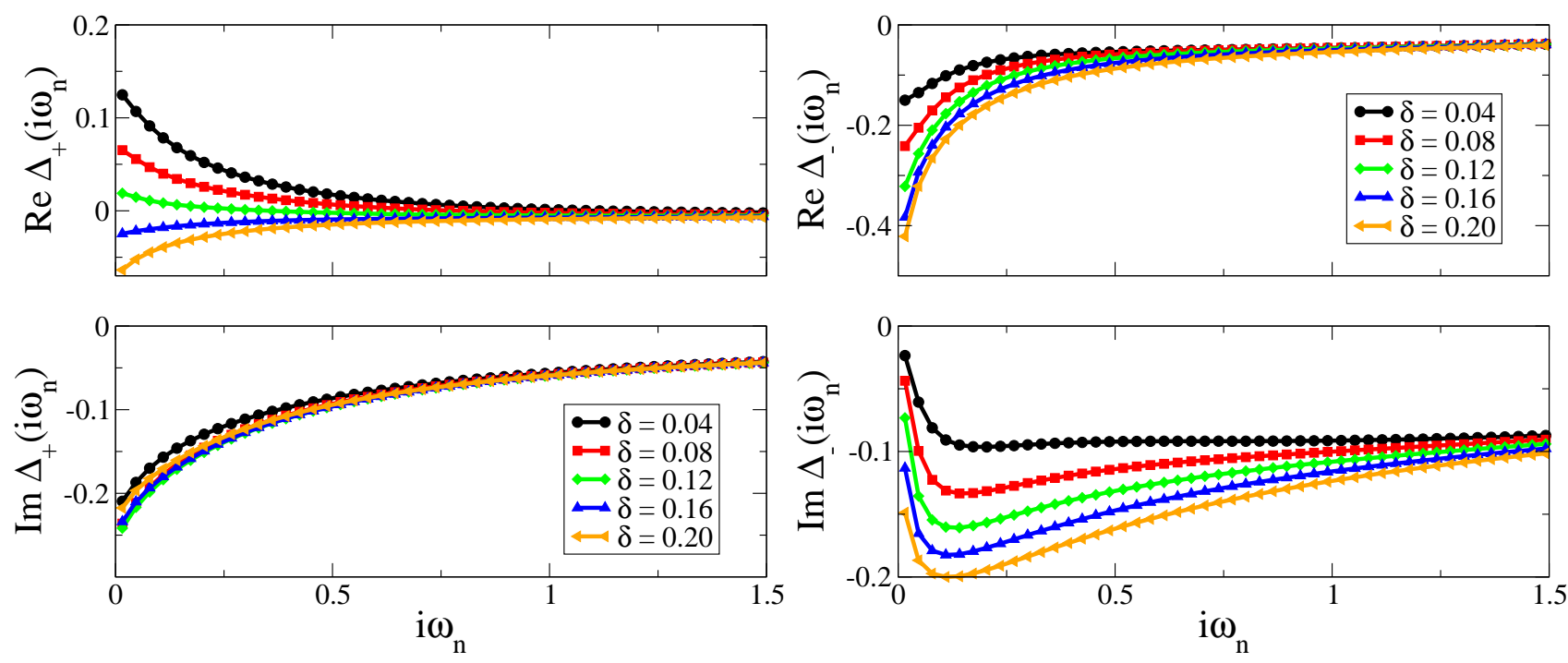

FIG. 27: (Color online) Even and odd hybridization functions $\Delta_{ \pm}\left(i \omega_{n}\right)$ on the Matsubara axis for various dopings at $\beta=200$.

49 C. Renner, B. Revaz, J.-Y. Genoud, K. Kadowaki, and O. Fischer, Phys. Rev. Lett. 80, 149 (1998).

50 A. Perali, M. Sindel, and G. Kotliar, epjb 24, 487 (2001).

${ }^{51}$ L. De Leo and M. Fabrizio, Phys. Rev. B 69, 245114 (2004).

${ }^{52}$ L. Zhu and C. M. Varma, Coherence in the two kondo impurity problem (2006), arXiv:cond-mat/0607426.

53 C. Jayaprakash, H. R. Krishnamurthy, and J. W. Wilkins, Phys. Rev. Lett. 47, 737 (1981).

54 B. A. Jones, B. G. Kotliar, and A. J. Millis, Phys. Rev. B 39, 3415 (1989).

${ }^{55}$ I. Affleck, A. W. W. Ludwig, and B. A. Jones, Phys. Rev. B 52, 9528 (1995).

56 P. S. Cornaglia and D. R. Grempel, Phys. Rev. B 71, 075305 (2005).

57 A. Houghton, N. Read, and H. Won, Phys. Rev. B 37, 3782 (1988).

58 T. A. Maier, T. Pruschke, and M. Jarrell, Phys. Rev. B 66, 075102 (2002).

59 T. D. Stanescu and G. Kotliar, Phys. Rev. B 74, 125110 (2006).

60 T. D. Stanescu, M. Civelli, K. Haule, and G. Kotliar, An- nals of Physics 321, 1682 (2006).

61 K. M. Shen, F. Ronning, D. H. Lu, F. Baumberger, N. J. C. Ingle, W. S. Lee, W. Meevasana, Y. Kohsaka, M. Azuma, M. Takano, et al., Science 307, 901 (2005).

62 F. H. L. Essler and A. M. Tsvelik, Phys. Rev. B 65, 115117 (2002).

63 I. Dzyaloshinskii, Phys. Rev. B 68, 085113 (2003).

${ }^{64}$ C. Berthod, T. Giamarchi, S. Biermann, and A. Georges, Phys. Rev. Lett. 97, 136401 (2006).

65 K.-Y. Yang, T. M. Rice, and F.-C. Zhang, Phys. Rev. B 73, 174501 (2006).

66 N. Doiron-Leyraud, C. Proust, D. LeBoeuf, J. Levallois, J.-B. Bonnemaison, R. Liang, D. A. Bonn, W. N. Hardy, and L. Taillefer, Nature 447, 565 (2007).

67 M. H. Hettler, A. N. Tahvildar-Zadeh, M. Jarrell, T. Pruschke, and H. R. Krishnamurthy, Phys. Rev. B 58, R7475 (1998).

68 M. H. Hettler, M. Mukherjee, M. Jarrell, and H. R. Krishnamurthy, Phys. Rev. B 61, 12739 (2000).

69 K. S. D. Beach, cond-mat/0403055.

70 A. W. Sandvik, Phys. Rev. B 57, 10287 (1998). 